\title{
ON SEMILATTICES OF GROUPS WHOSE ARROWS ARE EPIMORPHISMS
}

\author{
M. EL-GHALI M. ABDALLAH, L. N. GAB-ALLA, AND SAYED K. M. ELAGAN
}

Received 6 April 2006; Accepted 6 April 2006

A $q$ partial group is defined to be a partial group, that is, a strong semilattice of groups $S=\left[E(S) ; S_{e}, \varphi_{e, f}\right]$ such that $S$ has an identity 1 and $\varphi_{1, e}$ is an epimorphism for all $e \in E(S)$. Every partial group $S$ with identity contains a unique maximal $q$ partial group $\mathbf{Q}(S)$ such that $(\mathbf{Q}(S))_{1}=S_{1}$. This $\mathbf{Q}$ operation is proved to commute with Cartesian products and preserve normality. With $\mathbf{Q}$ extended to idempotent separating congruences on $S$, it is proved that $\mathbf{Q}\left(\rho_{K}\right)=\rho_{\mathbf{Q}(K)}$ for every normal $K$ in $S$. Proper $q$ partial groups are defined in such a way that associated to any group $G$, there is a proper $q$ partial group $\mathbf{P}(G)$ with $(\mathbf{P}(G))_{1}=G$. It is proved that a $q$ partial group $S$ is proper if and only if $S \cong \mathbf{P}\left(S_{1}\right)$ and hence that if $S$ is any partial group, there exists a group $M$ such that $S$ is embedded in $\mathbf{P}(M)$. $P$ epimorphisms of proper $q$ partial groups are defined with which the category of proper $q$ partial groups is proved to be equivalent to the category of groups and epimorphisms of groups.

Copyright (c) 2006 Hindawi Publishing Corporation. All rights reserved.

\section{Introduction and preliminaries}

One can easily observe that Clifford semigroups have been the object of extensive study from both category and semigroup theorists. A Clifford semigroup is usually defined as a regular semigroup with central idempotents. Whence many characterizations exist including the structure theorem that characterizes them as semilattices of groups, or equivalently as strong semilattices of groups. That is if $S=\left[Y, S_{\alpha}, \varphi_{\alpha, \beta}\right]$ is a strong semilattice $Y$ of groups $S_{\alpha}$, then $S$ is a Clifford semigroup with operation defined by

$$
a b=\left(\varphi_{\alpha, \alpha \beta} a\right)\left(\varphi_{\beta, \alpha \beta} b\right)
$$

for $a \in S_{\alpha}, b \in S_{\beta}$.

Conversely, a Clifford semigroup $S$ is a strong semilattice $E(S)$ of groups $S_{f} ; S=[E(S)$, $\left.S_{f}, \varphi_{f, g}\right]$ where $E(S)$ is the semilattice $(f \leq g \Leftrightarrow f g=f)$ of idempotents in $S, S_{f}$ is the maximal subgroup of $S$ with identity $f$, and $\varphi_{f, g}$ is the homomorphism $S_{f} \rightarrow S_{g}, a \mapsto a g$ if $f \geq g$. Here we observe that $S$ may be viewed as a category with objects all $S_{f}, f \in E(S)$

Hindawi Publishing Corporation

International Journal of Mathematics and Mathematical Sciences

Volume 2006, Article ID 30673, Pages 1-26

DOI 10.1155/IJMMS/2006/30673 
(are objects in the category of groups) and arrows, also called morphisms, given for two objects $S_{f}$ and $S_{g}$ as follows: $\operatorname{Hom}\left(S_{f}, S_{g}\right)=\left\{\varphi_{f, g}\right\}$ if $f \geq g$ and $\operatorname{Hom}\left(S_{f}, S_{g}\right)=\varnothing$ otherwise.

If $\alpha: S \rightarrow T$ is a homomorphism between Clifford semigroups, then clearly $f \geq g$ in $E(S)$ implies $\alpha(f) \geq \alpha(g)$ in $E(T)$. Also $\alpha\left(S_{f}\right) \subset T_{\alpha(f)}$. It follows that $\alpha$ is a functor between categories $S$ and $T$, that sends the object $S_{f}$ in $S$ to the object $T_{\alpha(f)}$ in $T$ and the arrow $\varphi_{f, g}$ in $S$ to the arrow $\varphi_{\alpha(f), \alpha(g)}$ in $T$.

In the present work we are concerned with those Clifford semigroups $S$ such that $S$ is also a monoid with identity $e$ and $\varphi_{e, f}: S_{e} \rightarrow S_{f}$ is an epimorphism for all $f \in E(S)$, that is, $S_{f}=S_{e} f$ for all $f \in E(S)$. We then call $S$ a $q$ partial group (or $q$ Clifford semigroup, etc.).

A partial group is defined in [3] as a semigroup $S$ such that every $x \in S$ has so-called a partial identity $e_{x}$ and a partial inverse $x^{-1}$ satisfying

(i) $e_{x} x=x e_{x}=x$ and if $y x=x y=x$, then $e_{x} y=y e_{x}=e_{x}$,

(ii) $x x^{-1}=x^{-1} x=e_{x}$ and $e_{x} x^{-1}=x^{-1} e_{x}=x^{-1}$,

(iii) $e_{x y}=e_{x} e_{y}$ and $(x y)^{-1}=y^{-1} x^{-1}$ for $x, y \in S$. That is the map $x \mapsto e_{x}$ is a homomorphism and $x \mapsto x^{-1}$ is antihomomorphism from $S$ into $S$.

It turns out that a partial group $S$ is precisely a strong semilattice of groups $\left[E(S), S_{f}\right.$, $\left.\varphi_{f, g}\right]$, that is, a Clifford semigroup. Also, it is proved in [3] that every partial group $S$ is embeddable in a partial group $\stackrel{\Delta}{P}(S, G)$ of partial mappings from $S$ to a suitable group $G$ (see Theorem 1.1). Such sort of structure and representation has been the basis for developing other kinds of "partial algebras" (see [1] for partial rings and [4] for partial monoids). Here in this section we will observe that any such $\stackrel{\triangle}{P}(S, G)$ is not just a partial group but also a $q$ partial group in the sense given above. Whence partial mappings (between sets and groups) may be considered as natural sources of $q$ partial groups.

In Section 2 we give some definitions and simple observations concerning $q$ partial groups. We introduce the $\mathbf{Q}$ operation in Section 3 and show that every partial group $S$ with identity contains a maximal $q$ partial group $\mathbf{Q}(S)$ which is a nontrivial if and only if $S_{1}$ is a nontrivial group, where $S_{1}$ is the maximal group in $S$ with identity $1_{S}$. This holds obviously for any wide (full) subpartial group $T$ of $S$. We show that the $\mathbf{Q}$ operation commutes with Cartesian products in partial groups with identities, and conclude that the product of any family of $q$ partial groups is a $q$ partial group. In Section 4 we extend the $\mathbf{Q}$ operation to the lattice $\mathbf{C}(S)$ of idempotent separating congruences $\rho$ on a partial group $S$ with identity and show that this operation preserves normality in $S$. We introduce the notion of a $q$ congruence on $S$ in such a way that for any $\rho \in \mathbf{C}^{\mathbf{i}}(S), \mathbf{Q}(\rho)$ is the maximal $q$ congruence contained in $\rho$ and that $\mathbf{Q}\left(\rho_{N}\right)=\rho_{\mathbf{Q}(N)}$, where $N=\operatorname{ker} \rho$. This allows to establish a complete modular lattice isomorphism between $q$ normal subpartial groups of $S$ and $q$ congruences on $S$. In Section 5 we are mainly concerned with those $q$ partial groups $S$ for which certain conditions are satisfied, and call them proper $q$ partial groups. We show that associated to any group $G$ there is a proper $q$ partial group $P(G)$, for which $(P(G))_{1}=G$, and give characterizations of different kinds of $q$ partial groups $S$ in terms of $P\left(S_{1}\right)$. This allows to embed arbitrary partial groups (i.e., Clifford semigroups) in proper $q$ partial groups of the kind $P(G)$. When morphisms in categories are restricted 
to epimorphisms in groups $(\mathbf{G})$ and to a certain kind of epimorphisms (called $p$ epimorphisms) in proper $q$ partial groups (PQP), we show in Section 6 that these two categories are equivalent. We also give certain characterizations of $p$ epimorphisms in $\mathbf{P Q P}$.

Almost all notations used throughout the paper are standard. Otherwise full explanations are accomplished. The notation $1_{S}$ (or sometimes just 1 ) is used either to denote the identity element of (the algebra) $S$ or the identity mapping $S \rightarrow S, x \mapsto x$. Its unique meaning in a definite situation is determined by the case analysis. Some notations (symbols) from logic may be used: for all, $\Rightarrow, \Leftrightarrow$ (in place of for all, implies, if and only if (iff), resp.). References for different topics are as follows:

(i) semigroups, in general: $[5,8]$;

(ii) groups: $[6,9]$;

(iii) categories: [7].

Notations, definitions, and results appeared in [2,3] and needed for our work are summarized here for the sake of reference. A subpartial group of a partial group $S$ is a subsemigroup $K$ of $S$ such that for all $x \in K, e_{x} \in K, x^{-1} \in K$. A subpartial group $K$ is wide (or full) if $E(S) \subset K$. If $S, T$ are partial groups, then $\alpha: S \rightarrow T$ is a homomorphism of partial groups if $\alpha(x y)=\alpha(x) \alpha(y)$ for all $x, y \in S$. Monomorphisms, epimorphisms, and so forth, and automorphisms are defined also as in semigroups. If $\alpha: S \rightarrow T$ is a homomorphism of partial groups, then $e_{\alpha(x)}=\alpha\left(e_{x}\right)$ and $(\alpha(x))^{-1}=\alpha\left(x^{-1}\right)$ for all $x \in S$. Thus $\alpha\left(S_{e}\right) \subset T_{\alpha(e)}$ for all $e \in E(S)$, where $S_{e}$ is the maximal group in $S$ with identity $e$. Also $\operatorname{Im} \alpha$ (range $\alpha$ ); that is, $\alpha(S)$ is a subpartial group of $T$.

If $X$ is a (nonempty) set and $G$ is a group, there is a partial group denoted $\stackrel{\triangle}{P}(X, G)$ whose elements are all partial mappings $f: X \longmapsto G$ (i.e., domain $f \subset X$ ) with multiplications defined as follows. For $f, g \in \stackrel{\Delta}{P}(X, G)$ say $f: A \rightarrow G, g: B \rightarrow G(A, B \subset X)$, $f g$ is the partial mapping $X \longmapsto G$ with domain $f g=\operatorname{dom} f \cap \operatorname{dom} g=A \cap B$ and $f g$ : $A \cap B \rightarrow G, x \mapsto f(x) g(x)$ where $f(x) g(x)$ is the multiplication in $G$. With this operation $\stackrel{\triangle}{P}(X, G)$ is a strong semilattice of groups (partial group) $\left[Y, M(A, G), \varphi_{A, B}\right]$, where $Y=\left\{e_{A}: e_{A}: A \rightarrow G, x \mapsto 1_{G}, A \subset X\right\}$ is the semilattice of idempotents in $\stackrel{\Delta}{P}(X, G)\left[e_{A} \leq\right.$ $e_{B}$ if and only if $\left.A \subset B\right], M(A, G)$ is the maximal group in $\stackrel{\triangle}{P}(X, G)$ with identity $e_{A}$, that is, the set of all mappings $f: A \rightarrow G$, and for $e_{A} \geq e_{B}$, that is, $B \subset A, \varphi_{e_{A, e_{B}}}$ (or simply $\varphi_{A, B}$ ) is the homomorphism $\varphi_{A, B}: M(A, G) \rightarrow M(B, G),\left.f \mapsto f\right|_{B}$ where $\left.f\right|_{B}: B \rightarrow G$ is the restriction of $f: A \rightarrow G$ on $B$. As we declared above, $\stackrel{\Delta}{P}(X, G)$ is actually a $q$ partial group. That is for all $B \subset X, \varphi_{X, B}: M(X, G) \rightarrow M(B, G)$ is an epimorphism of groups. For we have that $\stackrel{\triangle}{P}(X, G)$ has the identity element $e_{X}$ which is the identity of the maximal group $M(X, G)$, and given $f \in M(B, G)$, the mapping $\bar{f} \in M(X, G)$ defined by $\bar{f}(x)=f(x)$ if $x \in B$ and $\bar{f}(x)=1_{G}$ if $x \in X-B$ satisfies $\varphi_{X, B}(\bar{f})=f$. Thus $\varphi_{X, B}$ is an epimorphism.

Let $S$ be any partial group (i.e., Clifford semigroup). For each $x \in S$, define $S^{x}=\{y \in$ $\left.S: e_{x} y=y\right\}$ (called the $x$-ball in $\left.S[3]\right)$. We have $x, e_{x} \in S_{x} \subset S^{x}(\forall x \in S)$,

$S^{x}=S^{y}$ if and only if $e_{x}=e_{y}$,

$S^{x} \cap S^{y}=S^{x y}$.

Let $G$ be the coproduct of the maximal groups $S_{e_{x}}, e_{x} \in E(S)$, that is, $G=\amalg_{e_{x}} S_{e_{x}}$. Then for each $e_{x} \in E(S)$, there exists an injection (monomorphism) $i_{e_{x}}: S_{e_{x}} \rightarrow G$, satisfying the 
4 On semilattices of groups whose arrows are epimorphisms

desired universal property. For each $x \in S$, define

$$
\beta_{x}: S^{x} \longrightarrow G \quad \text { by } a \longmapsto i_{e_{a}}\left(x e_{a}\right)
$$

Thus $\beta_{x} \in \stackrel{\triangle}{P}(S, G)$ and $\beta_{x} \beta_{y}=\beta_{x y}$ for all $x, y \in S[3]$.

We have the following theorem.

Theorem 1.1 [3, the representation theorem]. Let $S, G$ be as above. There exists a monomorphism (embedding) of partial groups

$$
\alpha: S \longrightarrow \stackrel{\triangle}{P}(S, G)
$$

which sends each $x \in S$ to $\beta_{x} \in \stackrel{\triangle}{P}(S, G)$.

Let $S$ be a partial group. A subpartial group $K$ of $S$ is normal (denoted by $K \triangleleft S$ ) if $K$ is wide (i.e., $E(S) \subset K$ ) and $x K x^{-1} \subset K$ for all $x \in S$. Clearly $E(S)$ is normal in $S$ (it is the trivial normal subpartial group of $S$ ). We have the following proposition.

Proposition 1.2 [2]. If $K$ is a normal subpartial group of $S$, then $K_{e_{x}}$ is a normal subgroup of $S_{e_{x}}$ for all $x \in S$.

Let $\phi: S \rightarrow T$ be a homomorphism of partial groups. The $k$-kernel of $\phi$ (simply $k-$ $\operatorname{ker} \phi)$ is

$$
k-\operatorname{ker} \phi=\{x \in S: \phi(x)=e \text { for some } e \in E(T)\} .
$$

If $S$ is a partial group and $\rho$ is a congruence on $S$, then $S / \rho$ is a partial group called the quotient partial group induced by $\rho$. Moreover, $e_{x \rho}=e_{x} \rho$ and $(x \rho)^{-1}=x^{-1} \rho$ for all $x \in S$.

If $\phi: S \rightarrow T$ is a homomorphism of partial groups, then $\operatorname{ker} \phi=\{(x, y) \in S \times S: \phi(x)=$ $\phi(y)\}$ is an idempotent separating congruence on $S$ if and only if $\phi$ is idempotent separating.

Let $S$ be a partial group. Then, a congruence $\rho$ is idempotent separating if and only if $x \rho y \Rightarrow e_{x}=e_{y}$.

Associated to every congruence $\rho$ on $S$ there is a unique idempotent separating congruence on $S$ denoted by $\rho^{i}$, such that $x \rho^{i}=x \rho \cap S_{e_{x}}$ for all $x \in S$.

Theorem 1.3 [2]. Let $K$ be a normal subpartial group of S. Define

$$
\rho_{K}=\left\{(x, y) \in S \times S: e_{x}=e_{y} \text { and } x y^{-1} \in K\right\} .
$$

Then

(i) $\rho_{K}$ is an idempotent separating congruence on $S$ and $K=\operatorname{ker} \rho_{K}=k-\operatorname{ker}\left(\rho_{K}^{\sharp}\right)$, where $\rho_{K}^{\#}: S \rightarrow S / \rho_{K}$ is the canonical epimorphism,

(ii) $x \rho_{K}=x K_{e_{x}}$ for all $x \in S$,

(iii) $K=(E(S)) \rho_{K}=\cup\left\{e_{x} \rho_{K}: e_{x} \in E(S)\right\}$.

Theorem 1.4 [2]. For every idempotent separating congruence $\rho$ on $S$ there exists a normal subpartial group $K$ of $S$ with $K=\operatorname{ker} \rho=E(S) \rho$ and $\rho=\rho_{K}$. 
If $K$ is a normal subpartial group of a partial group $S$, the quotient partial group $S / \rho_{K}$ is denoted by $S / K$, where $\rho_{K}$ is the unique idempotent separating congruence on $S$ associated with $K$ (as in Theorem 1.4). We have the following.

Proposition $1.5[2] .(S / K)_{x K_{e_{x}}}=S_{e_{x}} / K_{e_{x}}$ for all $x \in S$.

Proposition 1.6 [2]. If $\phi: S \rightarrow T$ is a homomorphism of partial groups, then $\rho_{k-\operatorname{ker} \phi}=$ $(\operatorname{ker} \phi)^{i}$.

Theorem 1.7 [2]. Let $\phi: S \rightarrow T$ be a homomorphism of partial groups.

(a) There exists a unique homomorphism $\alpha: S / k-\operatorname{ker} \phi=S /(\operatorname{ker} \phi)^{i} \rightarrow T$ such that $\operatorname{ran}(\alpha)$ $=\operatorname{ran}(\phi)$ and the diagram

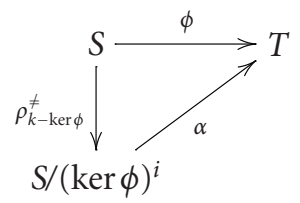

commutes.

(b) $\alpha$ is a monomorphism if and only if $\phi$ is idempotent separating.

(c) $\alpha$ is an isomorphism if and only if $\phi$ is both an epimorphism and idempotent separating.

Theorem 1.8 [2]. Let $\phi: S \rightarrow T$ be a homomorphism of partial groups. If $K$ is a normal subpartial group of $S$ such that $K \subset k-\operatorname{ker} \phi$, then there exists a unique homomorphism $\alpha: S / K \rightarrow T$ such that $\operatorname{ran}(\alpha)=\operatorname{ran}(\phi)$ and the diagram

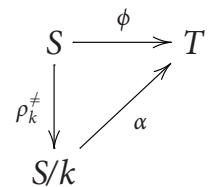

commutes.

Let $S$ be a partial group. The set of all idempotent separating congruence on $S$ is denoted by $\mathbf{C}^{\mathbf{i}}(S)$. We have $\mathbf{C}^{\mathbf{i}}(S)=\left\{\rho^{i}: \rho\right.$ is a congruence on $\left.S\right\}$, where $\rho^{i}$ is the maximal idempotent separating congruence on $S$ such that $\rho^{i} \subset \rho$. We have $\rho \circ \sigma=\sigma \circ \rho \in \mathbf{C}^{\mathbf{i}}(S)$ for all $\rho, \sigma \in \mathbf{C}^{\mathbf{i}}(S)$.

Proposition 1.9 [2]. $\left(\mathbf{C}^{\mathrm{i}}(S), \subset, \cap, \vee\right)$ is a complete modular lattice with $\rho \vee \sigma=\rho \circ \sigma$ for all $\rho, \sigma \in \mathbf{C}^{\mathbf{i}}(S)$.

The set of all normal subpartial groups of $S$ is denoted by $\mathbf{N}(S)$.

For $M, N \in \mathbf{N}(S), M N=N M$ is the minimal normal subpartial group of $S$ containing $M \cup N$, that is, $M N=N M=\langle M \cup N\rangle$ is the (normal) subpartial group of $S$ generated by $M \cup N$ or the join $M \vee N$ of $M$ and $N$.

Proposition 1.10 [2]. If $M, N \in \mathbf{N}(S)$, then $\rho_{M \cap N}=\rho_{M} \cap \rho_{N} ; \rho_{M N}=\rho_{M} \circ \rho_{N}$. 
Theorem $1.11[2] .(\mathrm{N}(S), \subset, \cap, \vee)$ is a complete modular lattice and the mapping

$$
\varphi: \mathbf{N}(S) \longrightarrow \mathbf{C}^{\mathbf{i}}(S), \quad K \longmapsto \rho_{K}
$$

is a lattice isomorphism.

\section{2. $q$ partial groups}

Throughout this section, $S$ stands for a partial group with identity 1 . Thus $S$ is a strong semilattice of groups $\left[E(S) ; S_{f}, \varphi_{f, g}\right]$ with $E(S)$ having upper bound 1 . We call the identity 1 of $S$ proper if the maximal subgroup $S_{1}$ is not the trivial group, that is, if $\{1\}$ is a proper subset of $S_{1}$. Otherwise, 1 is called improper. In the usual partial ordering of $E(S)$, we then have $1 \geq e$ for all $e \in E(S)$, and so we have a homomorphism of groups $\varphi_{1, e}: S_{1} \rightarrow S_{e}$ for every $e \in E(S)$.

We call $S$ a q partial group if $\varphi_{1, e}$ is an epimorphism for every $e \in E(S)$. This is equivalent to say that $S_{1} e=S_{e}$ for every $e \in E(S)$, that is, every $x \in S_{e}$ can be written as a product $y e$ for some $y \in S_{1}$. Since $S_{1} e \subset S_{e}$ always holds, $S$ is a $q$ partial group if and only if $S_{e} \subset S_{1} e$ for all $e \in E(S)$.

If $S$ is $q$ partial group and $e \geq f$ in $E(S)$, we then have $\varphi_{e, f}\left(S_{e}\right)=S_{e} f=\left(S_{1} e\right) f=$ $S_{1}(e f)=S_{1} f=S_{f}$. It follows that in a $q$ partial group $S$, every homomorphism $\varphi_{e, f}$ is an epimorphism. We observe also that in a $q$ partial group $S, S_{e} \cdot S_{f}=S_{e f}$ for all $e, f \in E(S)$. For, we have $S_{e} \cdot S_{f} \subset S_{e f}$ since $S$ is a strong semilattice of its maximal subgroups, and if $x \in S_{e f}$, then $x=y e f$ for some $y \in S_{1}$, which gives $x=(y e)(1 f) \in S_{e} \cdot S_{f}$.

Let $T$ be a wide subpartial group of $S$. Then we can call $T$ a $q$ subpartial group of $S$ if the restriction $\varphi_{1, e}$ on $T_{1}$ is an epimorphism for every $e \in E(S)$, that is, $T$ is a $q$ subpartial group with the inherited operations from $S$. Trivially, every semilattice with upper bound is a $q$ partial group, and hence $E(S)$ is a $q$ subpartial group of $S$. If the identity 1 of $S$ is improper, we clearly have $S=E(S)$, and so $S$ reduces to a semilattice. The converse holds trivially. Thus for any $q$ partial group, we have $S \neq E(S)$ if and only if 1 is proper, that is, if and only if $S_{1}$ is not the trivial group. As we observed in the introduction, the partial group $\stackrel{\triangle}{P}(X, G)$, for any set $X$ and group $G$, is a $q$ partial group with identity $1_{X}: X \rightarrow G$, $x \mapsto 1_{G}$, the identity $1_{X}$ is the identity of the maximal group $M(X, G)$ in $\stackrel{\Delta}{P}(X, G)$. Clearly $M(X, G)$ is not the trivial group if and only if $X$ is nonempty and $G$ is not the trivial group. Thus $\stackrel{\triangle}{P}(X, G)$ is a nontrivial $q$ partial group if and only if $X \neq \varnothing$ and $G \neq 0$. We close this section by one more simple observation.

LEMMA 2.1. If $S$ is a q partial group in which no two maximal subgroups are isomorphic, then the kernels of the epimorphisms $\varphi_{1, e}, e \in E(S)$, are all different.

Proof. Let $N_{e}$ denote the kernel of $\varphi_{1, e}, e \in E(S)$. Then $N_{e}=\left\{y \in S_{1}: y e=e\right\}$. If $e \neq f$ in $E(S)$ and $N_{e}=N_{f}$, we have by the first isomorphism theorem of groups

$$
S_{e} \cong S_{1} / N_{e}=S_{1} / N_{f} \cong S_{f}
$$

which contradicts the hypothesis. 


\section{The Q operation}

In the previous section we noticed that every partial group $S$ with identity contains a trivial $q$ subpartial group, namely, $E(S)$. In this section, we show that nontrivial $q$ subpartial groups of $S$ exist whenever $S_{1}$ is a nontrivial group. More precisely, a maximal $q$ subpartial group of $S$ always exists. This inherited to all wide subpartial groups $T$ of $S$, and hence defines an operation $T \rightarrow \mathbf{Q}(T)$. In later work, we will show that the $\mathbf{Q}$ operation preserves normality, and commutes with the operation of taking joins. In this section, we show that it commutes with categorical products. Given a wide subpartial groups $T$ of $S$, the existence of $\mathbf{Q}(T)$, the maximal $q$ subpartial group contained in $T$ can be verified by the axiom of choice (e.g., Zorn's lemma), but for later purpose we construct $\mathbf{Q}(T)$ explicitly. It is obtained simply by taking images of $\varphi_{1, e}$ on $T_{1}$ for all $e \in E(S)$. Formally, we have the following lemma.

Lemma 3.1. Let $S$ be a partial group with identity and let $T$ be a wide subpartial group of $S$. There exists a q subpartial group $\mathbf{Q}(T)$ of $S$ which is unique maximal such that $\mathbf{Q}(T) \subset T$. Moreover $\mathbf{Q}(T)$ is nontrivial (i.e., does not equal $E(S)$ ) if and only if $T_{1}$ is a nontrivial group.

Proof. Since $T$ is wide, it is a union of maximal groups indexed by $E(S)$, that is, $T=$ $\left[E(S), T_{e}, \varphi_{e, f}\right]$ where $T_{e}$ is a subgroup of $S_{e}$ and $\varphi_{1, e}: T_{1} \rightarrow T_{e}$ is a homomorphism $(x \mapsto$ $x e)$ for every $e \in E(S)$. Define

$$
\mathbf{Q}(T)=\bigcup_{e \in E(S)} \operatorname{Im} \varphi_{1, e}=\bigcup_{e \in E(S)} T_{1} e .
$$

$\mathbf{Q}(T)$ is a disjoint union of groups $(\mathbf{Q}(T))_{e}=T_{1} e$ indexed by the semilattice $E(S)$. In particular, $(\mathbf{Q}(T))_{1}=\operatorname{Im} \varphi_{1,1}=T_{1}$ and the restriction of $\varphi_{1, e}$ on $T_{1}$ gives an epimorphism $\varphi_{1, e}:(\mathbf{Q}(T))_{1}=T_{1} \rightarrow(\mathbf{Q}(T))_{e}$ for every $e \in E(S)$.

It follows that $\mathbf{Q}(T)$ is $q$ subpartial group of $S$ contained in $T$.

For $e>f, \varphi_{e, f}: T_{1} e \rightarrow T_{1} f$ is given by $x e \mapsto x f,\left(x \in T_{1}\right)$.

If $K$ is $q$ subpartial group of $S$ with $K \subset T$, then $K_{1} \subset T_{1}=(\mathbf{Q}(T))_{1}$ and for all $e \in E(S)$, $K_{e}=K_{1} e \subset T_{1} e=(\mathbf{Q}(T))_{e}$. This proves the unique maximality of $\mathbf{Q}(T)$.

Finally, $\mathbf{Q}(T) \neq E(S) \Rightarrow(\mathbf{Q}(T))_{e} \neq\{e\}$ for some $e \in E(S) \Rightarrow T_{1} e \neq\{e\} \Rightarrow T_{1} \neq\{1\}$, conversely, if $T_{1} \neq\{1\}$, then $(\mathbf{Q}(T))_{1}=T_{1} \neq\{1\}$, and so $\mathbf{Q}(T) \neq E(S)$.

Let us now consider partial groups as a part of universal algebra, that is as a variety of algebras (defined by a set of identities). This implies that, as a category, partial groups have all small limits and colimits (e.g., products, coproducts, etc.). This is also true for partial groups with identities. In the rest of this section we consider categorical products of partial groups (with identities) and show that the $\mathbf{Q}$ operation commutes with this product which implies that product of any family of $q$ partial groups is again a $q$ partial group. We start by characterizing products in the category of partial groups.

Lemma 3.2. Let $\left\{S_{i}, i \in I\right\}$ be a family of partial groups and let $S=\prod_{i \in I} S_{i}$ be the usual Cartesian product. Then $S$ is a partial group which is a categorical product with the usual projections $\pi_{i}: S \rightarrow S_{i}\left(x_{i}\right) \mapsto x_{i}$. If each $S_{i}$ has an identity $1_{s_{i}}$, then $\left(1_{s_{i}}\right)$ is the identity of $S$. 
8 On semilattices of groups whose arrows are epimorphisms

Proof. Define $E(S)=\prod_{i \in I} E\left(S_{i}\right)$. Then $E(S)$ is a semilattice with $\left(e_{i}\right) \leq\left(f_{i}\right)$ if and only if $e_{i} \leq f_{i}$, for all $i \in I$.

We have

$$
S=\prod_{i \in I} S_{i}=\prod_{i \in I} \bigcup_{e_{i} \in E\left(S_{i}\right)}\left(S_{i}\right)_{e_{i}}=\bigcup_{\left(e_{i}\right) \in E(S)} \prod_{i \in I}\left(S_{i}\right)_{e_{i}} .
$$

Thus $S$ is a disjoint union of groups $\prod_{i \in I}\left(S_{i}\right)_{e_{i}}, e_{i} \in E\left(S_{i}\right)$ with identities $\left(e_{i}\right)_{i \in I}, e_{i} \in E\left(S_{i}\right)$, indexed by the semilattice $E(S)$. For $\left(e_{i}\right) \geq\left(f_{i}\right)$ in $E(S)$, there is a homomorphism

$$
\varphi_{\left(e_{i}\right),\left(f_{i}\right)}: \prod_{i \in I}\left(S_{i}\right)_{e_{i}} \longrightarrow \prod_{i \in I}\left(S_{i}\right)_{f_{i}},
$$

given by

$$
\left(x_{i}\right) \longmapsto\left(\varphi_{e_{i}, f_{i}} x_{i}\right) .
$$

Now we can easily verify that $S$ is a categorical product, and that $S$ has identity if each $S_{i}$ has a one.

Theorem 3.3. Let $\left\{S_{i}, i \in I\right\}$ be a family of partial groups, with identities. Then $\mathbf{Q}\left(\prod_{i \in I} S_{i}\right)$ $=\prod_{i \in I} \mathbf{Q}\left(S_{i}\right)$.

Proof. By Lemma 3.2, $\prod_{i \in I} S_{i}$ is a partial group with identity $\left(1_{s_{i}}\right)_{i \in I}$ which is a union of maximal subgroups $\left(\prod_{i \in I} S_{i}\right)_{\left(e_{i}\right)}$ indexed by the semilattice $E(S)=\prod_{i \in I} E\left(S_{i}\right)$. By Lemma 3.1, we have

$$
\begin{aligned}
\mathbf{Q}\left(\prod_{i \in I} S_{i}\right) & =\bigcup_{\left(e_{i}\right) \in E(S)}\left(\prod_{i \in I} S_{i}\right)_{\left(1_{s_{i}}\right)}\left(e_{i}\right) \\
& =\bigcup_{\left(e_{i}\right) \in E(S)}\left(\prod_{i \in I}\left(S_{i}\right)_{1_{s_{i}}} e_{i}\right)=\prod_{i \in I}\left(\bigcup_{e_{i} \in E\left(S_{i}\right)}\left(S_{i}\right)_{1_{s_{i}}} e_{i}\right) \\
& =\bigcup_{\left(e_{i}\right) \in E(S)}\left(\prod_{i \in I}\left(S_{i}\right)_{1_{s_{i}}} e_{i}\right)=\prod_{i \in I} \mathbf{Q}\left(S_{i}\right) .
\end{aligned}
$$

By the definition of the $\mathbf{Q}$ operation, one can show that the product of any family of $q$ partial groups is again a $q$ partial group. But if we notice that for any partial group $S$ with identity, $S$ is a $q$ partial group if and only if $\mathbf{Q}(S)=S$, then the following is an easy consequence of Theorem 3.3.

Corollary 3.4. If $\left\{S_{i}, i \in I\right\}$ is a family of $q$ partial groups, then the product $\prod_{i \in I} S_{i}$ is a $q$ partial group.

\section{Normality and $q$ congruences}

In this section we develop certain properties of the $\mathbf{Q}$ operation needed for further work. We show it preserves normality in partial groups with identities. The notation of a $q$ normal subpartial group will play an important role in later work, so we introduce the notion 
of a $q$ congruence on a partial group $S$ with identity in such a way that $\rho$ is a $q$ congruence on $S$ if and only if $\operatorname{ker} \rho$ is a $q$ normal subpartial group of $S$. For any idempotent separating congruence $\rho$ on $S$ we define $\mathbf{Q}(\rho)$ and show it is the maximal $q$ congruence contained in $\rho$. This extends the $\mathbf{Q}$ operation to the lattice of all idempotent separating congruences on $S$, with the property that if $S$ is a $q$ partial group, then for any normal subpartial group $N$ of $S, \mathbf{Q}(N)$ is normal in $S$ and $\mathbf{Q}\left(\rho_{N}\right)=\rho_{\mathbf{Q}(N)}$. This allows to establish a complete modular lattice isomorphism between $q$ normal subpartial groups of $S$ and $q$ congruence on $S$, which is analogous to the classical result (see, e.g., [2]) known for idempotent separating congruences.

We begin by a technical lemma showing that the join of any family of $q$ subpartial groups is again a $q$ subpartial group. First, we need to recall some preliminaries.

Remark 4.1. If $S$ is any partial group and $X$ is any (nonempty) subset of $S$, the subpartial group $\langle X\rangle$ generated by $X$ is the intersection of all subpartial groups of $S$ containing $X$. Actually, $\langle X\rangle$ is the set of all finite products $x_{i_{1}}^{n_{1}} x_{i_{2}}^{n_{2}} \cdots x_{i_{k}}^{n_{k}}$ with $x_{i j} \in X, n_{j}= \pm 1$. If $X \cap S_{e} \neq \varnothing$ for all $e \in E(S)$, then $\langle X\rangle$ is also wide. The elements of $X$ are the generators of $\langle X\rangle$. If $s \in\langle X\rangle$, say $s \in(\langle X\rangle)_{e}$ for some $e \in E(S)$ (i.e., $\left.e_{s}=e\right)$, then $s$ may be represented by a finite product

$$
s=x_{i_{1}} x_{i_{2}} \cdots x_{i_{k}},
$$

with $x_{i j} \in S_{e}$ for all $j=1, \ldots, k$, (just multiply each generator in the typical expansion of $s$ by $e$ ).

Lemma 4.2. Let $S$ be a partial group with identity and let $\left\{S_{i}, i \in I\right\}$ be a family of $q$ subpartial groups of $S$. Then $\left\langle\bigcup_{i \in I} S_{i}\right\rangle$ is a q subpartial group of $S$.

Proof. Clearly $\left\langle\bigcup_{i \in I} S_{i}\right\rangle$ is a wide subpartial group of $S$. Let $e \in E(S)$ and let $s \in\left(\left\langle\bigcup_{i \in I} S_{i}\right\rangle\right)_{e}$. By the above remark (and since each $S_{i}$ is a subpartial group of $S$ ), we have

$$
s=x_{i_{1}} x_{i_{2}} \cdots x_{i_{k}}
$$

with $x_{i j} \in\left(S_{i j}\right)_{e}, j=1, \ldots, k$.

Since $S_{i j}$ is a $q$ subpartial group of $S$,

$$
\left(S_{i j}\right)_{e}=\left(S_{i j}\right)_{1} e, \quad j=1, \ldots, k .
$$

Therefore

$$
s=\left(s_{1} s_{2} \cdots s_{k}\right) e \in\left(\left\langle\bigcup_{i \in I} s_{i}\right\rangle\right)_{1} e .
$$

This gives

$$
\left(\left\langle\bigcup_{i \in I} S_{i}\right\rangle\right)_{e}=\left(\left\langle\bigcup_{i \in I} S_{i}\right\rangle\right)_{1} e
$$

and the result obtains. 
Now we show that the $\mathbf{Q}$ operation commutes with the join operation on wide subpartial groups.

Lemma 4.3. Let $S$ be as in Lemma 4.2, and let $\left\{S_{i}, i \in I\right\}$ be a family of wide subpartial groups of $S$. Then $\mathbf{Q}\left(\left\langle\bigcup_{i \in I} S_{i}\right\rangle\right)=\left\langle\bigcup_{i \in I} \mathbf{Q}\left(S_{i}\right)\right\rangle$.

Proof. By Lemma 4.2, $\left\langle\bigcup_{i \in I} \mathbf{Q}\left(S_{i}\right)\right\rangle$ is a q subpartial group of $S$, which is clearly contained in $\left\langle\bigcup_{i \in I} S_{i}\right\rangle$. Thus it is sufficient to show that it is maximal with respect to this property. For this, let $T$ be a qubpartial group of $S$ with $T \subset\left\langle\bigcup_{i \in I} S_{i}\right\rangle$, and let $t \in T$, say $t \in$ $T_{e}=T_{1} e$, for some $e \in E(S)$. Thus $t=s e$, for some $s \in T_{1}$, and since $T_{1} \subset\left(\left\langle\bigcup_{i \in I} S_{i}\right\rangle\right)_{1}$, we obtain

$$
s=x_{i_{1}} x_{i_{2}} \cdots x_{i_{k}},
$$

with $x_{i j} \in\left(S_{i j}\right)_{1}, j=1, \ldots, k$.

This gives

$$
\begin{aligned}
t & =\left(x_{i_{1}} x_{i_{2}} \cdots x_{i_{k}}\right) e \\
& =\left(x_{i_{1}} e\right) \cdots\left(x_{i_{k}} e\right) \in\left(\mathbf{Q}\left(S_{i_{1}}\right)\right)_{e} \cdots\left(\mathbf{Q}\left(S_{i_{k}}\right)\right)_{e}, \quad \text { since }\left(S_{i j}\right)_{1} e=\left(\mathbf{Q}\left(S_{i_{j}}\right)\right)_{e} .
\end{aligned}
$$

Therefore

$$
t \in\left\langle\bigcup_{i \in I} \mathbf{Q}\left(S_{i}\right)\right\rangle .
$$

This proves maximality, and the proof is complete.

Given a partial group $S$ with identity, then clearly, a normal subpartial group of $S$ need not be a $q$ subpartial group. For a trivial example, take $S$ such that $1_{s}$ is improper, and $S \neq E(S)$. Then $S$ is normal in $S$ but not a $q$ partial group. This is also the case even if $S$ is a $q$ partial group, for example, take $S$ such that $1_{s}$ is proper, $S_{e} \neq\{e\}$, for some $e \in E(S)$ and let $T<S$ be such that $T_{1}=\left\{1_{s}\right\}$ and $T_{e}=S_{e}$ otherwise. On the other hand, the $\mathbf{Q}$ operation preserves normality in $q$ partial groups. This is our next result.

LEMma 4.4. Let $S$ be a q partial group if $N$ is a normal subpartial group of $S$, so is $\mathbf{Q}(N)$.

Proof. Let $s \in S, x \in \mathbf{Q}(N)$, say $s \in S_{f}$ and $x \in(\mathbf{Q}(N))_{g}$, for some $f, g \in E(S)$.

We have

$$
(\mathbf{Q}(N))_{g}=(\mathbf{Q}(N))_{1} g
$$

and so $x=y g$, for some $y \in(\mathbf{Q}(N))_{1}$. By the definition of the $\mathbf{Q}$ operation and the normality of $N$, we have

$$
y \in(\mathbf{Q}(N))_{1}=N_{1} \triangleleft S_{1} .
$$

Also $s \in S_{f}=S_{1} f$, say, $s=s_{1} f$ for some $s_{1} \in S_{1}$. 
Thus

$$
s^{-1} x s=\left(s_{1} f\right)^{-1}(y g)\left(s_{1} f\right)=\left(s_{1}^{-1} y s\right)(f g) \in N_{1} f g=(\mathbf{Q}(N))_{f g} \subset \mathbf{Q}(N) .
$$

Before introducing $q$ congruences on a partial group $S$ with identity, we consider the set of all $q$ normal subpartial groups of $S$ and show it is a lattice whenever $S$ is a $q$ partial group. We know, in the lattice $\mathbf{N}$ of all normal subpartial groups of a partial group $S$, the join and meet are given by $M \vee N=M N=\langle M \cup N\rangle$ and $M \wedge N=M \cap N$, respectively. For $q$ normal subpartial groups $M, N$, if $S$ has an identity, then $\langle M \cup N\rangle$ is already a $q$ normal subpartial group of $S$ (Lemma 4.2). However, $M \cap N$ need not be a $q$ (normal) subpartial group of $S$, even if $S$ is a $q$ partial group, because the homomorphism $\varphi_{1, e}$ need not be one-to-one for arbitrary $e \in E(S)$. Here is a simple example.

Example 4.5. Let $X=\{a, b\}$ and $G=Z_{2}=\{0,1\}$, and let $S=P(X, G)$ be the corresponding $q$ partial group of partial mappings (see Sections 1,2).

Then $P(X, G)$ is the union of 4 maximal groups, $\stackrel{\triangle}{P}(S, G)=M(X, G) \cup M(\{a\}, G) \cup$ $M(\{b\}, G) \cup\{z\}$, where $z$ represents the empty mapping $\phi \rightarrow G$, and is the zero element of $\stackrel{\Delta}{P}(S, G)$. Let $T_{1}=\{(a, b) \mapsto(0,0),(a, b) \mapsto(1,1)\}$. Then $T_{1}$ is a subgroup of $M(X, G)$ consists of just two mappings.

Let $R_{1}=\{(a, b) \mapsto(0,0),(a, b) \mapsto(1,0)\}$. Let $e_{a}$ be the mapping $\{a\} \rightarrow G, a \mapsto 0$, and let $e_{b}$ be the mapping $\{b\} \rightarrow G, b \mapsto 0$.

Denoting $T_{1} e_{a}$ by $T_{a}$, and so forth, we have

$$
\begin{aligned}
& T_{a}=T_{1} e_{a}=\{a \mapsto 0, a \mapsto 1\}, \\
& T_{b}=T_{1} e_{b}=\{b \mapsto 0, b \mapsto 1\}, \\
& T_{z}=T_{1} z=\{z\}, \\
& R_{a}=R_{1} e_{a}=\{a \mapsto 0, a \mapsto 1\}, \\
& R_{b}=R_{1} e_{b}=\{b \mapsto 0, b \mapsto 1\}, \\
& R_{z}=R_{1} z=\{z\} .
\end{aligned}
$$

Let $T=T_{1} \cup T_{a} \cup T_{b} \cup T_{z}$, and $R=R_{1} \cup R_{a} \cup R_{b} \cup R_{z}$. With the induced operation from $S$, both $T$ and $R$ are $q$ normal subpartial groups of $S$.

But $T \cap R=\{(a, b) \mapsto(0,0)\} \cup\{a \mapsto 0, a \mapsto 1\} \cup\{b \mapsto 0, b \mapsto 1\} \cup\{z\}$ is a (normal) subpartial group of $S$, which is not a $q$ subpartial group.

Let $S$ be a $q$ partial group and let $\mathrm{QN}(S)$ denote the set of all $q$ normal subpartial groups of $S$. In view of Lemmas 3.1, 4.2, and 4.4, we easily obtain the following result.

LEMMA 4.6. $\mathrm{QN}(S)$ is a complete modular lattice with meet and join defined by

$$
M \wedge N=\mathbf{Q}(M \cap N), \quad M \vee N=M N=\langle M \cup N\rangle .
$$

The notion of a $q$ normal subpartial group of a partial group $S$ with identity is nothing but an idempotent separating congruence on $S$ that satisfies certain condition. Here we give the definition.

Given a partial group $S$ with identity, we call an idempotent separating congruence $\rho$ on $S$ a $q$ congruence on $S$ if for all $x, y \in S, x \rho y$ implies $x=s y$, for some $s \in(\operatorname{ker} \rho)_{1}$, that is, for some $s \in S_{1}$ with $s \rho 1$. 
We observe that, if $\rho$ is $q$ congruence, then $x \rho y$ implies (also) $x=y s$, for some $s \rho 1$.

The following consequence follows at once from the definition. (Recall that $\rho_{K}$ is the congruence whose kernel is $K$.)

Lemma 4.7. Let $S$ be a partial group with identity, and let $\rho$ be an idempotent separating congruence on $S$. Then $\rho$ is q congruence if and only if $K=\operatorname{ker} \rho$ is a q normal subpartial group of $S$. Equivalently, for any subpartial group $K$ of $S, K$ is a $q$ normal subpartial group of $S$ if and only if $\rho_{K}$ is a $q$ congruence.

Let $S$ be a partial group with identity and $\mathbf{C}^{\mathbf{i}}(S)$ the lattice of all idempotent separating congruences on $S$. Recall that, for $\rho, \sigma \in \mathbf{C}^{\mathbf{i}}(S)$, the join and meet are given by $\rho \circ \sigma=$ $\sigma \circ \rho=\rho \vee \sigma$ (e.g., [2], Lemma 5.1) and $\rho \wedge \sigma=\rho \cap \sigma$, respectively.

Again, the intersection of two $q$ congruences on $S$ needs not be a $q$ congruence (e.g., apply Lemma 4.7 to Example 4.5). So we define a $\mathbf{Q}$ operation on $\rho \in \mathbf{C}^{\mathbf{i}}(S)$ as follows:

$$
\mathbf{Q}(\rho)=\{(x, y): x \rho y, x=s y(\text { or } x=y s) \text {, for some } s \in S \text { with } s \rho 1\} .
$$

Equivalently, $\mathbf{Q}(\rho)$ is the unique maximal $q$ congruence on $S$ contained in $\rho$.

Lemma 4.8. Let $S$ be a q partial group and let $\rho \in \mathbf{C}^{\mathbf{i}}(S)$. Then,

$$
\mathbf{Q}(\rho)=\rho_{\mathbf{Q}(N)}, \quad \text { where } N=\operatorname{ker} \rho .
$$

Proof. By definition, $N=\{x \in S: x \rho e$ for some $e \in E(S)\}$, and $\rho=\rho_{N}$. By Lemma 4.4, $\mathbf{Q}(N)$ is normal in $S$. Now, let $(x, y) \in \mathbf{Q}(\rho)=\mathbf{Q}\left(\rho_{N}\right)$. Then $x \rho y$ and $x=s y$, for some $s \in S$ with $s \rho 1$, that is, for some $s \in(\operatorname{ker} \rho)_{1}=N_{1}$. We have $e_{x}=e_{y}$ (since $\rho$ is idempotent separating), and $x y^{-1} \in N_{e_{x}}$.

By definition of the $\mathbf{Q}$ operation

$$
(\mathbf{Q}(N))_{e_{x}}=N_{1} e_{x}
$$

and so

$$
x y^{-1}=(s y) y^{-1}=s e_{x} \in N_{1} e_{x} \subset \mathbf{Q}(N),
$$

whence

$$
(x, y) \in \rho_{\mathbf{Q}(N)} .
$$

Thus

$$
\mathbf{Q}(\rho) \subset \rho_{\mathbf{Q}(N)} .
$$

Conversely, let $(x, y) \in \rho_{\mathbf{Q}(N)}$. Thus

$$
e_{x}=e_{y}, \quad x y^{-1} \in(\mathbf{Q}(N))_{e_{x}}=N_{1} e_{x} .
$$

That is

$$
x y^{-1}=s e_{x} \quad \text { for some } s \in N_{1} \text {. }
$$


Thus

$$
x y^{-1} \in N, \quad x=s y \text { for some } s \in N_{1}=(\operatorname{ker} \rho)_{1},
$$

and so

$$
(x, y) \in \mathbf{Q}(\rho)=\mathbf{Q}\left(\rho_{N}\right) .
$$

Therefore

$$
\mathbf{Q}(\rho)=\mathbf{Q}\left(\rho_{N}\right)=\rho_{\mathbf{Q}(N)} .
$$

For a $q$ partial group $S$, let $\mathbf{Q} \mathbf{C}^{\mathbf{i}}(S)$ denote the set of all $q$ congruences on $S$. We have the following theorem.

Theorem 4.9. $\mathrm{QC}^{\mathrm{i}}(S)$ is a complete modular lattice, with meet and join given by

$$
\begin{gathered}
\rho \wedge \sigma=\mathbf{Q}(\rho \cap \sigma), \\
\rho \circ \sigma=\sigma \circ \rho=\rho \vee \sigma, \quad \text { respectively. }
\end{gathered}
$$

Moreover, the mapping

$$
\varphi: \mathbf{Q N}(S) \longrightarrow \mathbf{Q C}^{\mathbf{i}}(S), \quad N \longmapsto \rho_{N}
$$

is a lattice isomorphism.

Proof. It is easy to see that $\rho \circ \sigma \in \mathbf{Q C}^{\mathbf{i}}(S)$, if $\rho, \sigma \in \mathbf{Q} \mathbf{C}^{\mathbf{i}}(S)$, and that $\mathbf{Q C}^{\mathbf{i}}(S)$ is a lattice. The mapping $\varphi$ is well defined by Lemma 4.7, and clearly is one-to-one and onto. By Lemmas 4.6 and 4.8 , we have for $N, M \in \mathrm{QN}(S)$

$$
\begin{aligned}
\varphi(N \wedge M) & =\varphi(\mathbf{Q}(N \cap M))=\rho_{\mathbf{Q}(N \cap M)}=\mathbf{Q}\left(\rho_{N \cap M}\right) \\
& =\mathbf{Q}\left(\rho_{N} \cap \rho_{M}\right)=\rho_{N} \wedge \rho_{M}=\varphi(N) \wedge \varphi(M) .
\end{aligned}
$$

Likewise,

$$
\varphi(N \vee M)=\varphi(N) \vee \varphi(M)
$$

\section{Developing certain representations}

Given a $q$ partial group $S$, there is an isomorphism of groups $S_{1} / N_{e} \cong S_{e}$, for every $e \in$ $E(S)$, where $N_{e}=\operatorname{ker} \varphi_{1, e}$. On the other hand, given a (nonzero) group $G$, we can (and do) generate a $q$ partial group $P(G)$, such that $(P(G))_{1}=G$ and every maximal group in $\mathrm{P}(G)$ is a quotient $G / N$, for some normal $N$ in $G$. In this section, we develop the connection between particular types of $q$ partial groups $S$, and their associated $q$ partial groups $\mathrm{P}\left(S_{1}\right)$.

Naturally, partial groups of partial mappings $\stackrel{\Delta}{P}(X, G)$ are $q$ partial groups. This allows to 
embed arbitrary partial groups (i.e., Clifford semigroups) in a $q$ partial groups of the kind $P(G)$.

Let $G$ be a (nontrivial) group and let $\mathbf{N}$ be the set of all normal subgroups of $G$. We define a partial ordering $\leq$ on $\mathbf{N}$ by

$$
M \leq N \Longleftrightarrow N \subset M .
$$

We have a semilattice $\langle\mathbf{N}, \leq, \wedge\rangle$ with $M \wedge N=\langle M \cup N\rangle=M N$, for all $N, M \in \mathbf{N}$.

$\mathbf{N}$ has an upper bound $1_{G}$ (and lower bound $\{G\}$ ). If $\mathbf{N}$ is indexed by a set $\mathbf{I}$, we have a bijection

$$
\mathbf{I} \longrightarrow \mathbf{N}, \quad i \longmapsto N_{i}
$$

This bijection is a semilattice isomorphism if we define $\leq$ on $\mathbf{I}$ by $i \leq j$ if and only if $N_{i} \leq N_{j}$; if and only if $N_{j} \subset N_{i}$, for all $i, j \in \mathrm{I}$. $i \wedge j=i j=k$ if and only if $N_{i} N_{j}=N_{k}$. In this case, we have the following lemma.

Lemma 5.1. Let $G$ be a group, and let $N=\left\{N_{i}: i \in \mathbf{I}\right\}$ be the semilattice of all normal subgroups of $G$ defined as above. Let

$$
P(G)=\bigcup_{i \in \mathbf{I}} G / N_{i}
$$

Then $P(G)$ is a partial group with multiplication

$$
x N_{i} \cdot y N_{j}=x y N_{i} N_{j}=x y N_{i j},
$$

identity element $1=1_{G}$, and zero element $z=G / G$.

Proof. For $N_{i} \geq N_{j}$ (equivalently, $i \geq j, N_{i} \subset N_{j}$ ), define

$$
\varphi_{i, j}: G / N_{i} \longrightarrow G / N_{j}, \quad x N_{i} \longmapsto x N_{j} .
$$

We have $x_{1} N_{i}=x_{2} N_{i} \Rightarrow x_{1} x_{2}^{-1} \in N_{i} \subset N_{j} \Rightarrow x_{1} N_{j}=x_{2} N_{j}$, and hence $\varphi_{i, j}$ is well defined.

For $x_{1} N_{i}, x_{2} N_{i} \in G / N_{i}$

$$
\begin{aligned}
\varphi_{i, j}\left(x_{1} N_{i} x_{2} N_{i}\right) & =\varphi_{i, j}\left(x_{1} x_{2} N_{i}\right)=x_{1} x_{2} N_{j} \\
& =x_{1} N_{j} x_{2} N_{j}=\varphi_{i, j}\left(x_{1} N_{i}\right) \varphi_{i, j}\left(x_{2} N_{i}\right) .
\end{aligned}
$$

Thus $\varphi_{i, j}$ is a homomorphism of groups which is clearly an epimorphism. Identifying $i \in \mathbf{I}$ with the identity of $G / N_{i}$ (for all $i \in \mathbf{I}$ ), we have for all $i \in \mathbf{I}$ an epimorphism

$$
\varphi_{1, i}: G /\left\{1_{G}\right\}=G \longrightarrow G / N_{i}, \quad x \longmapsto x N_{i} .
$$

Clearly, $\varphi_{i, i}$ is the identical automorphism, and $\varphi_{j, k} \circ \varphi_{i, j}=\varphi_{i, k}$, for every $i \geq j \geq k$ in $\mathbf{I}$. Thus $P(G)$ is a $q$ partial group with

$$
x N_{i} y N_{j}=\varphi_{i, i j} x N_{i} \cdot \varphi_{j, i j} y N_{j}=x N_{i j} y N_{i j}=x y N_{i j}=x y N_{i} N_{j} .
$$

The identity 1 of $P(G)$ is the identity of the maximal group $(P(G))_{1}=G /\left\{1_{G}\right\}=G$ which is $1_{G}$, and it has a zero $z=$ (the identity of) $G / G$. 
Our next technicality holds for any arbitrary partial group.

Lemma 5.2. Let $S$ be a partial group, and let $\left\{T_{e^{\prime}}\right\}$ be a family of (pairwise disjoint) groups indexed by a semilattice $\left\{e^{\prime}: e \in E(S)\right\}$ isomorphic to $E(S)$, such that corresponding groups are isomorphic. Then $T=\cup T_{e^{\prime}}$ is a partial group isomorphic to $S$.

Proof. We have a semilattice of groups

$$
S=\left[E(S), S_{e}, \varphi_{e, f}\right] .
$$

Let $E(T)$ denote the semilattice $\left\{e^{\prime}: e \in E(S)\right\}$ isomorphic to $E(S)$, with $e \mapsto e^{\prime}$. For each $e \in E(S)$, there exists an isomorphism of groups

$$
\alpha_{e}: S_{e} \stackrel{\cong}{\longrightarrow} T_{e^{\prime}} .
$$

For $e^{\prime} \geq f^{\prime}$ in $E(T), e \geq f$ in $E(S)$, and we define

$$
\varphi_{e^{\prime}, f^{\prime}}: T_{e^{\prime}} \longrightarrow T_{f^{\prime}}
$$

by

$$
\varphi_{e^{\prime}, f^{\prime}}=\alpha_{f} \varphi_{e, f} \alpha_{e}^{-1} .
$$

It is easily seen that $\varphi_{e^{\prime}, f^{\prime}}$ is a well-defined epimorphism of groups, that $\varphi_{e^{\prime}, e^{\prime}}$ is the identical automorphism of $T_{e^{\prime}}$, and that $\varphi_{f^{\prime}, g^{\prime}} \cdot \varphi_{e^{\prime}, f^{\prime}}=\varphi_{e^{\prime}, g^{\prime}}$ for all $e^{\prime} \geq f^{\prime} \geq g^{\prime}$ in $E(T)$. In other words, $T$ is a semilattice $E(T)$ of groups $T_{e^{\prime}}$, and we identify $e^{\prime}$ with the identity of $T_{e^{\prime}}$ for each $e^{\prime} \in E(T)$. Thus $E(T)$ is the semilattice of idempotents in $T$. We now extend the isomorphism

$$
E(S) \longrightarrow E(T), \quad e \longmapsto e^{\prime}
$$

to an isomorphism $S \rightarrow T$. Define $\alpha: S \rightarrow T$ as follows: for any $x \in S$, say $x=x_{e} \in S_{e}$ for some $e \in E(S)$, set

$$
\alpha\left(x_{e}\right)=\alpha_{e}\left(x_{e}\right)
$$

Thus $\alpha$ is a well-defined mapping (since $S_{e}, e \in E(S)$ are pairwise disjoint) with restriction, an isomorphism $\alpha_{e}: S_{e} \rightarrow T_{e^{\prime}}$, for each $e \in E(S)$. For arbitrary $x, y \in S$, say, $x=x_{e} \in$ $S_{e}, y=y_{f} \in S_{f}$, we have $x y \in S_{e f}$. Thus

$$
\alpha(x y)=\alpha\left(x_{e} y_{f}\right)=\alpha\left(x_{e} y_{f} e f\right)=\alpha_{e f}\left(x_{e} y_{f} e f\right) .
$$

Using $(*)$, and since $e \mapsto e^{\prime}$ is isomorphism, we also have

$$
\begin{aligned}
\alpha(x) \alpha(y) & =\alpha\left(x_{e}\right) \alpha\left(y_{f}\right)=\alpha_{e}\left(x_{e}\right) \alpha_{f}\left(y_{f}\right) \\
& =\varphi_{e^{\prime}, e^{\prime} f^{\prime}}\left(\alpha_{e}\left(x_{e}\right)\right) \circ \varphi_{f^{\prime}, e^{\prime} f^{\prime}}\left(\alpha_{f}\left(y_{f}\right)\right) \\
& =\alpha_{e f}\left(\varphi_{e, e f} \alpha_{e}^{-1}\left(\alpha_{e}\left(x_{e}\right)\right)\right) \circ \alpha_{e f}\left(\varphi_{f, e f} \alpha_{f}^{-1}\left(\alpha_{f}\left(y_{f}\right)\right)\right) \\
& =\alpha_{e f}\left(\varphi_{e, e f}\left(x_{e}\right)\right) \circ \alpha_{e f}\left(\varphi_{f, e f}\left(y_{f}\right)\right) \\
& =\alpha_{e f}\left(\varphi_{e, e f}\left(x_{e}\right) \varphi_{f, e f}\left(y_{f}\right)\right)=\alpha_{e f}\left(x_{e} y_{f} e f\right) .
\end{aligned}
$$


It follows that $\alpha(x y)=\alpha(x) \alpha(y)$. Thus $\alpha$ is a homomorphism of partial groups which is clearly one-to-one, onto $T$, and the proof is complete.

Now we discuss particular kinds of $q$ partial groups.

Let $S$ be a $q$ partial group and let $N_{e}$ be the kernel of $\varphi_{1, e}, e \in E(S)$. Let $S$ satisfy the condition

$$
e \neq f \quad \text { in } E(S) \Longrightarrow N_{e} \neq N_{f}
$$

Thus

$$
E(S) \longrightarrow \mathbf{N}=\left\{N_{e}: e \in E(S)\right\}, \quad e \longmapsto N_{e}
$$

is a bijection, which is an isomorphism of semilattices, where $\leq$ is defined on $\mathbf{N}$ by

$$
N_{e} \leq N_{f} \Longleftrightarrow e \leq f,
$$

which gives for all $e, f \in E(S)$

$$
N_{e} \wedge N_{f}=N_{e f}
$$

Here we observe

$$
N_{e} \leq N_{f} \Longrightarrow N_{f} \subset N_{e},
$$

equivalently, $e \leq f \Rightarrow N_{f} \subset N_{e}$, (for we have $x \in N_{f} \Rightarrow x f=f \Rightarrow x e=x(e f)=(x f) e=$ $e \Rightarrow x \in N_{e}$ ).

Lemma 2.1 gives a sufficient condition for (5.16), that is a $q$ partial group $S$ satisfies (5.16) if the maximal groups in $S$ are pairwise nonisomorphic.

Remark 5.3. Partial mappings provide concrete examples for $q$ partial groups satisfying (5.16).

Let $X$ be a nonempty set, $G$ a nontrivial group, and let

$$
S=\stackrel{\triangle}{P}(X, G)
$$

be the corresponding $q$ partial group of partial mappings (cf. Section 1). We have that $S_{1}=M(X, G)$ is the group of all mappings from $X$ into $G$, and

$$
E(S)=\left\{e_{A}: A \longrightarrow G, x \longmapsto 1_{G} ; A \subset X\right\}
$$

is the semilattice with $e_{A} \leq e_{B}$ if and only if $A \subset B$. For all $e_{A}, e_{B} \in E(S)$, we also have

$$
e_{A} \wedge e_{B}=e_{A \cap B} .
$$

The identity 1 of $S$ is the identity of $S_{1}$, that is, $1_{S}$ is the mapping $e_{X}: X \rightarrow G, x \mapsto 1_{G}$, (here $1_{G}$ is the identity element of the group $\left.G\right)$. For all $A \subset X$,

$$
\varphi_{1, e_{A}}: M(X, G) \longrightarrow M(A, G)
$$


is (the epimorphism) given by $\left.f \mapsto f\right|_{A}$, (the restriction of $f$ on $A$ ). Thus,

$$
\begin{aligned}
N_{e_{A}} & =\operatorname{ker} \varphi_{1, e_{A}}=\left\{f \in M(X, G): f e_{A}=e_{A}\right\} \\
& =\left\{f \in M(X, G):\left.f\right|_{A}=e_{A}\right\} .
\end{aligned}
$$

Evidently, for all $A, B \subset X$

$$
A=B \Longleftrightarrow e_{A}=e_{B} .
$$

Now for all $A, B \subset X, N_{e_{A}}=N_{e_{B}} \Rightarrow$ for all $f \in M(X, G),\left.f\right|_{A}=e_{A}$ if and only if $\left.f\right|_{B}=e_{B} \Leftrightarrow$ $A=B \Leftrightarrow e_{A}=e_{B}$. It follows that

$$
e_{A} \neq e_{B} \Longrightarrow N_{e_{A}} \neq N_{e_{B}}
$$

as we claimed.

LEMMA 5.4. Let $S$ be a q partial group satisfying (5.16), and let $\mathbf{N}$ be the semilattice isomorphic to $E(S)$ given by (5.17). Then

$$
T=\bigcup_{e \in E(S)} S_{1} / N_{e}
$$

is a partial group, and

$$
\alpha: S \longrightarrow T, \quad x e \longmapsto x N_{e}
$$

is a partial group isomorphism.

Proof. For each $e \in E(S)$, there exists an isomorphism

$$
\theta_{e}: S_{1} / N_{e} \longrightarrow S_{e}
$$

given by

$$
\theta_{e}\left(x N_{e}\right)=\varphi_{1, e} x, \quad \forall x \in S_{1} .
$$

That is,

$$
\theta_{e}\left(x N_{e}\right)=x e .
$$

Observe that $\varphi_{1, e}$ is an epimorphism, and so every element in $S_{e}$ is $x e$, for some $x \in S_{1}$. Thus for every $e \in E(S)$, we have an isomorphism

$$
\alpha_{e}: S_{e} \longrightarrow S_{1} / N_{e}
$$

given by

$$
\alpha_{e}=\theta_{e}^{-1}, \quad \text { that is } \alpha_{e}(x e)=x N_{e}
$$


Applying Lemma 5.2 (with $N_{e}=e^{\prime}$ ), it follows that $T$ is a partial group with homomorphisms

$$
\left.\varphi_{N_{e}, N_{f}}=\alpha_{f} \varphi_{e, f} \alpha_{e}^{-1}: S_{1} / N_{e} \longrightarrow S_{1} / N_{f}, \quad \forall N_{e} \geq N_{f} \text { (i.e., } e \geq f \text { in } E(S)\right) .
$$

Thus, for arbitrary $x N_{e} \in S_{1} / N_{e}$,

$$
\varphi_{N_{e}, N_{f}}\left(x N_{e}\right)=\alpha_{f} \varphi_{e, f} \alpha_{e}^{-1}\left(x N_{e}\right)=x N_{f} .
$$

Actually, $\varphi_{N_{e}, N_{f}}$ is an epimorphism. Using (5.19), multiplication in $T$ is given as follows: for arbitrary $x N_{e}, y N_{f} \in T\left(x, y \in S_{1}\right)$

$$
x N_{e} \cdot y N_{f}=\varphi_{N_{e}, N_{e f}} x N_{e} \cdot \varphi_{N_{f}, N_{e f}} y N_{f}=x N_{e f} \cdot y N_{e f}=x y N_{e f} .
$$

Lemma 5.5. Let $\mathrm{S}, \mathbf{N}$ be as in Lemma 5.4. Then,

$$
\forall e, f \in E(S), \quad N_{e} \subset N_{f} \Longrightarrow e \geq f
$$

if and only if

$$
\forall e, f \in E(S), \quad N_{e f}=\left\langle N_{e} \cup N_{f}\right\rangle=N_{e} N_{f} .
$$

Proof. Suppose (5.38) holds. Then, by using (5.20), we obtain for any $e, f \in E(S)$

$$
e \geq f \Longleftrightarrow N_{e} \geq N_{f} \Longleftrightarrow N_{e} \subset N_{f}
$$

Thus the partial ordering of $\mathbf{N}$ is completely given by set inclusion in a reverse manner. Since $\left\langle N_{e} \cup N_{f}\right\rangle=N_{e} N_{f}\left(=N_{f} N_{e}\right.$ ) is the least upper bound of $N_{e}$ and $N_{f}$ with respect to $C$, it follows that, it is the greatest lower bound of them with respect to $\leq$ in $\mathbf{N}$. That is $N_{e} \wedge N_{f}=N_{e} N_{f}$. This and (5.19) give (5.39).

Conversely, suppose that (5.39) holds, and let $N_{e} \subset N_{f}(e, f \in E(S))$. Then

$$
N_{e f}=N_{e} N_{f}=N_{f},
$$

which gives, by (5.16), $e f=f$, and so $e \geq f$.

Let $S$ be a $q$ partial group satisfying (5.16) and the equivalent conditions (5.38) and (5.39). We call $S$ proper if also

$$
\text { for } N \subset S_{1}, \quad N \text { is normal in } S_{1} \text { only if } N=N_{e} \text { for some } e \in E(S) \text {, }
$$

(recall that $N_{e}=\operatorname{ker} \varphi_{1, e}$ ). By (5.16), if $S$ is proper and $N \triangleleft S_{1}$, then $N=N_{e}$ for a unique $e \in E(S)$.

A typical example of a proper $q$ partial group is the $q$ partial group $P(G)$ associated to a (nonzero) group $G$ (as in Lemma 5.1). Partial groups in general and (proper) $q$ partial groups in particular can be represented by proper $q$ partial groups of the kind $P(G)$. We devote the rest of this section for such representations. 
Theorem 5.6. Every q partial group $S$ that satisfies (5.16) and the equivalent conditions (5.38) and (5.39) is embeddable in its associated proper q partial group $P\left(S_{1}\right)$.

Proof. By Lemma 5.4, there exists an isomorphism

$$
\alpha: S \longrightarrow T, \quad x e \longmapsto x N_{e}
$$

where

$$
T=\bigcup_{e \in E(S)} S_{1} / N_{e}
$$

and $N_{e} \leq N_{f}$ if and only if $e \leq f$. Using (5.38), we obtain

$$
N_{e} \leq N_{f} \Longleftrightarrow N_{f} \subset N_{e}
$$

It follows that $E(T)$ is isomorphic to a subsemilattice of $E\left(P\left(S_{1}\right)\right)$. Since each maximal group $S_{1} / N_{e}$ is a maximal group in $P\left(S_{1}\right)$ (with identity $N_{e}$ ), it follows by Lemma 5.2 that $T$ is a subpartial group of $P\left(S_{1}\right)$. Hence $\alpha$ embeds $S$ in $P\left(S_{1}\right)$.

Corollary 5.7. Let $S$ be an arbitrary partial group and let $M$ be the group $M(S, G)$ of all mappings from $S$ into $G$, where $G$ is the coproduct of all maximal groups in $S$. Then $S$ is embeddable in $P(M)$.

Proof. By the representation theorem (Section 1), there exists a monomorphism

$$
\beta_{1}: S \longrightarrow \stackrel{\triangle}{P}(S, G)
$$

The partial group $\stackrel{\Delta}{P}(S, G)$ satisfies (5.16) (see the previous remark), and it is easy to see that all these partial groups (of partial mappings) satisfy (5.38) as well. Hence by Theorem 5.6, there exists an embedding

$$
\beta_{2}: \stackrel{P}{P}(S, G) \longrightarrow P(\stackrel{\triangle}{P}(S, G))_{1}=P(M(S, G))
$$

$\beta_{2} \beta_{1}$ is the required embedding.

The proof of the following lemma is routine and omitted.

Lemma 5.8. Let $S$ and $T$ be partial groups with identities, and let $\gamma: S \rightarrow T$ be a homomorphism.

(i) If $S$ is a q partial group and $\gamma$ maps $E(S)$ onto $E(T)$, then $\operatorname{Im} \gamma$ is a q subpartial group of $T$. In particular, if $\gamma$ is an epimorphism, then $T$ is a $q$ partial group.

(ii) If $\gamma$ is an isomorphism, then $T$ is proper if (and only if) $S$ is proper.

Corollary 5.9. A q partial group $S$ is proper if and only if $S$ is isomorphic to $P\left(S_{1}\right)$.

Proof. Sufficiency follows from Lemma 5.8(ii). Necessity is a special case of Theorem 5.6 with $T=P\left(S_{1}\right)$. 
Nonzero groups and nontrivial commutative bands with identities are obvious examples of $q$ partial groups which are not proper. Eventhough, it is true that $q$ partial groups that satisfy (5.16) and (5.38) (equivalently (5.39)) need not be proper. A simple example is given by a group $G$ that has a proper normal subgroup $N$. For instance, let $S=S_{1} \cup S_{2}$ with indexed semilattice $1>2$, where $S_{1}=Z_{6}$ and $S_{2}=Z_{6} /\{0,3\}$. Then $S$ is a $q$ partial group satisfying (5.16) and (5.38) but does not satisfy (5.42). This can also be illustrated by partial mappings.

Example 5.10. Let $X$ be a nonempty set and let $G$ be as above, that is, $1_{G} \neq G$ is a group with a normal subgroup $1_{G} \neq N \neq G$, (e.g., $G=Z_{6}$ ). Let

$$
T=\{f \in M(X, G): f(X) \subset N\}
$$

clearly $T$ is a normal subgroup of $M(X, G)=\stackrel{\triangle}{P}(X, G)_{1}$. Also $T \neq M(X, G)$, since $N \neq G$. Let $A \subset X$ be fixed but arbitrary. We have (by definition)

$$
N_{e_{A}}=\operatorname{ker} \varphi_{e_{X}, e_{A}}=\left\{f \in M(X, G):\left.f\right|_{A}=e_{A}\right\} .
$$

Recall that $e_{A}$ is the identity of the maximal group $M(A, G)$, that is, $e_{A}: A \rightarrow G, x \mapsto 1_{G}$. Thus

$$
N_{e_{A}}=\left\{f: X \rightarrow G: f(A)=\left\{1_{G}\right\}\right\} .
$$

If $A=\varnothing$, then $N_{e_{A}}=M(X, G) \neq T$.

If $A \neq \varnothing$, choose $a \in A, p \in N$ and define $g: X \rightarrow G$ by

$$
x \longmapsto \begin{cases}p & \text { if } x=a, \\ 1_{G} & \text { if } x \neq a .\end{cases}
$$

Then $g \in T$, but $g \notin N_{e_{A}}$. Thus $T \neq N_{e_{A}}$. Since $A$ is arbitrary, it follows that $T \neq$ $\operatorname{ker} \varphi_{1, e_{A}}$, for every idempotent $e_{A}$ in $\stackrel{\triangle}{P}(X, G)$. Hence condition (5.42) does not hold, that is $\stackrel{\triangle}{P}(X, G)$ is not proper.

A proper $q$ partial group $S$ has (up to an isomorphism) a "local" quotient group $S_{1} / N_{e}$ at every point $e \in E(S)$. These can be integrated into a whole quotient partial group describing $S$. Formally, we have the following theorem.

THEOREM 5.11. Every proper q partial group $S$ is isomorphic to some quotient partial group $T / N$, with $N \neq E(T)$ if $1_{S}$ is proper. Explicitly, $T$ is a strong semilattice $E(S)$ of isomorphic copies of $S_{1}$.

Proof. By assumption, the set of all normal subgroups of $S_{1}$ is (the semilattice)

$$
\mathbf{N}=\left\{N_{e}=\operatorname{ker} \varphi_{1, e}: e \in E(S)\right\},
$$

with

$$
e \geq f \Longleftrightarrow N_{e} \geq N_{f} \Longleftrightarrow N_{e} \subset N_{f} .
$$


Let $\left\{T_{e}\right\}_{e \in E(S)}$ be disjoint isomorphic copies of $S_{1}$, with isomorphisms of groups

$$
\alpha_{e}: S_{1} \stackrel{\cong}{\longrightarrow} T_{e} \quad \text { for every } e \in E(S) .
$$

Let $T=\bigcup_{e \in E(S)} T_{e}$. For all $e \geq f$ in $E(S)$,

$$
\varphi_{e, f}^{\prime}: T_{e} \longrightarrow T_{f}
$$

given by

$$
\varphi_{e, f}^{\prime}=\alpha_{f} \alpha_{e}^{-1},
$$

clearly induce a semilattice of groups $T=\left[E(S) ; T_{e}, \varphi_{e, f}^{\prime}\right]$. For each $e \in E(S)$, there exists an isomorphism

$$
\gamma_{e}: S_{1} / N_{e} \longrightarrow T_{e} / N_{e}^{\prime}, \quad x N_{e} \longmapsto \alpha_{e}(x) N_{e}^{\prime},
$$

where $N_{e}^{\prime}$ is the image of $N_{e}$ under $\alpha_{e}$. Define $N_{e}^{\prime} \geq N_{f}^{\prime}$ if and only if $e \geq f$ in $E(S)$. Let $N=\bigcup_{e \in E(S)} N_{e}^{\prime}$. Then $N$ is a normal subpartial group of $T$ and the quotient partial group $T / N$ has maximal groups

$$
(T / N)_{e}=T_{e} / N_{e} \cong S_{1} / N_{e} \quad(e \in E(S)) .
$$

By Lemma 5.2, T/N is isomorphic to $P\left(S_{1}\right)$, whence by Corollary $5.9, S$ is isomorphic to $T / N$. The other assertion of the theorem follows immediately.

\section{Two equivalent categories}

We use previous results to show that proper $q$ partial groups together with certain kind of epimorphisms are a category equivalent to the category of groups and epimorphisms of groups.

Lemma 6.1. If $\alpha: S \rightarrow T$ is an epimorphism of $q$ partial groups, then the restriction $\left.\alpha\right|_{S_{1}}$ on $S_{1}$ is an epimorphism $S_{1} \rightarrow T_{1}$ of groups.

Proof. $\alpha\left(1_{S}\right)=e^{\prime}$ for some $e^{\prime} \in E(T)$. Since $\alpha$ is epimorphism, $\alpha(e)=1_{T}$ for some $e \in$ $E(S)$. Thus

$$
1_{T}=\alpha(e)=\alpha\left(1_{S} e\right)=\alpha\left(1_{S}\right) \alpha(e)=\alpha\left(1_{S}\right) 1_{T}=\alpha\left(1_{S}\right) .
$$

It follows that $\alpha\left(S_{1}\right) \subset T_{1}$.

If $y \in T_{1}$, there exists $s \in S$, say $s \in S_{e}$ for some $e \in E(S)$, such that $\alpha(s)=y$. Since $S$ is a $q$ partial group, $s=x e$ for some $x \in S_{1}$. Thus

$$
1_{T}=e_{y}=e_{\alpha(s)}=\alpha\left(e_{s}\right)=\alpha(e) .
$$

Therefore

$$
y=\alpha(s)=\alpha(x) \alpha(e)=\alpha(x) 1_{T}=\alpha(x),
$$

whence $y \in \alpha\left(S_{1}\right)$. The result follows. 
From now on, we use symbols to denote terms as follows: groups in general (are denoted by): $G, H, K, \ldots$,

(proper) $q$ partial groups: $S, T, R, \ldots$,

epimorphisms of $q$ partial groups: $\bar{\alpha}, \bar{\beta}, \bar{\gamma}, \ldots$,

restrictions of epimorphisms $\bar{\alpha}: S \rightarrow T, \bar{\beta}, \ldots$, of proper $q$ partial groups on maximal groups $S_{1}, \ldots$, are denoted by $\alpha: S_{1} \rightarrow T_{1}, \beta, \ldots$, (see Lemma 6.1).

Given a group $G$,

$$
\mathbf{N}(G)=\{N \subset G: N \triangleleft G\}
$$

For a $q$ partial group $S$,

$$
\mathbf{N}\left(S_{1}\right)=\left\{N \subset S_{1}: N \triangleleft S_{1}\right\} .
$$

In particular, if $S$ is a proper $q$ partial group,

$$
\mathbf{N}\left(S_{1}\right)=\left\{N_{e}=\operatorname{ker} \varphi_{1, e}: e \in E(S)\right\} .
$$

Unless stated otherwise, $\mathbf{N}(G)$ is a semilattice with $M \leq N$ if and only if $N \subset M$.

In particular, if $S$ is a proper $q$ partial group, $\mathbf{N}(S)$ is the semilattice for which

$$
e \leq f \Longleftrightarrow N_{e} \leq N_{f} \Longleftrightarrow N_{f} \subset N_{e} \quad(\forall e, f \in E(S)) \text { (cf. Section 5). }
$$

We call an epimorphism $\bar{\alpha}: S \rightarrow T$ (of proper $q$ partial groups) a $p$ epimorphism if

$$
\alpha\left(N_{e}\right)=N_{\bar{\alpha}(e)} \quad \text { for every } e \in E(S)
$$

In other words, $\bar{\alpha}$ is $p$ epimorphism if

$$
\alpha\left(\operatorname{ker} \varphi_{1, e}\right)=\operatorname{ker} \varphi_{1, \bar{\alpha}(e)} \quad \text { for every } e \in E(S)
$$

The next result which gives a characterization of $p$ epimorphisms is independent of later work. First we recall some definitions. A partially ordered set $(P, \leq)$ is said to satisfy the maximal condition if any nonempty subset of $P$ contains a maximal element. This is equivalent to say that $P$ satisfies the ascending chain condition: there does not exist an infinite properly ascending chain $x_{1}<x_{2}<\ldots$, in $P$.

The minimal condition and the descending chain condition are defined dually. A group $G$ satisfies the maximal condition on normal subgroups if $\langle\mathbf{N}(G), \subset\rangle$ satisfies the maximal condition (the partial ordering here is set inclusion). This property is identical with the ascending chain condition on the normal subgroups. Minimal and descending chain conditions on normal subgroups are defined dually.

As we mentioned above, where $\mathbf{N}(G)$ ( or $\mathbf{N}(S)$ ) is written without any further mention of partial ordering, we always mean the semilattice $(\mathbf{N}(G), \leq), M \leq N$ if and only if $N \subset$ $M$. Otherwise the partial ordering is written explicitly. Thus, for instance, if $S$ is a proper $q$ partial group, then $E(S)$ satisfies the maximal condition if and only if $\mathbf{N}\left(S_{1}\right)$ satisfies the maximal condition; if and only if $\left(\mathbf{N}\left(S_{1}\right), \subset\right)$ satisfies the minimal condition; if and only 
if $S_{1}$ satisfies the minimal condition on normal subgroups; if and only if there does not exist an infinite properly descending chain $N_{1} \supset N_{2} \supset \cdots$ in $\mathbf{N}\left(S_{1}\right)$.

Lemma 6.2. Let $S, T$ be proper $q$ partial groups and let $\bar{\alpha}: S \rightarrow T$ be an epimorphism.

(i) If $\bar{\alpha}$ is $p$ epimorphism, then the following condition holds:

$$
\bar{\alpha}(e)=\bar{\alpha}(f) \Longleftrightarrow \alpha\left(N_{e}\right)=\alpha\left(N_{f}\right) \quad(e, f \in E(S)) .
$$

(ii) If (6.10) holds and $T_{1}$ satisfies the maximal condition on normal subgroups (i.e., $E(T)$ satisfies the minimal condition), then $\bar{\alpha}$ is p epimorphism.

Proof. (i) Trivial.

(ii) Let $e_{0} \in E(S)$ be fixed but arbitrary. Clearly, $\alpha\left(N_{e_{0}}\right) \subset N_{\bar{\alpha}\left(e_{0}\right)}\left(x \in N_{e_{0}} \Rightarrow x e_{0}=e_{0} \Rightarrow\right.$ $\left.\bar{\alpha}(x) \bar{\alpha}\left(e_{0}\right)=\bar{\alpha}\left(e_{0}\right) \Rightarrow \alpha(x) \in N_{\bar{\alpha}\left(e_{0}\right)}\right)$. If $\alpha\left(N_{e_{0}}\right)=N_{\bar{\alpha}\left(e_{0}\right)}$ the result obtains. Otherwise, we have $N_{\bar{\alpha}\left(e_{0}\right)} \triangleleft T_{1} \Rightarrow N_{\bar{\alpha}\left(e_{0}\right)}=\alpha\left(N_{e_{1}}\right) \subset N_{\bar{\alpha}\left(e_{1}\right)}$ for some $e_{1} \in E(S)$ (since $S, T$ are proper and $\alpha$ is epimorphism). If $N_{\bar{\alpha}\left(e_{0}\right)}=N_{\bar{\alpha}\left(e_{1}\right)}$, then $\bar{\alpha}\left(e_{0}\right)=\bar{\alpha}\left(e_{1}\right)$ (by condition (5.16)) and so by (6.10), $\alpha\left(N_{e_{0}}\right)=\alpha\left(N_{e_{1}}\right)=N_{\bar{\alpha}\left(e_{0}\right)}$ and the result follows. Otherwise and having chosen $e_{k}$, there exists $e_{k+1} \in E(S)$ with $N_{\bar{\alpha}\left(e_{k}\right)}=\alpha\left(N_{e_{k+1}}\right)$ and so $N_{\bar{\alpha}\left(e_{k}\right)} \subset N_{\bar{\alpha}\left(e_{k+1}\right)}$. This would give an infinite chain $N_{\bar{\alpha}\left(e_{0}\right)} \subset N_{\bar{\alpha}\left(e_{1}\right)} \subset \cdots \subset N_{\bar{\alpha}\left(e_{k}\right)} \subset N_{\bar{\alpha}\left(e_{k+1}\right)} \subset \cdots$.

By the maximal condition, we must have $N_{\bar{\alpha}\left(e_{j}\right)}=N_{\bar{\alpha}\left(e_{j+1}\right)}$ for some $j \geq 1$. Thus $\bar{\alpha}\left(e_{j}\right)=$ $\bar{\alpha}\left(e_{j+1}\right)$, which gives by (6.10), $\alpha\left(N_{e_{j}}\right)=\alpha\left(N_{e_{j+1}}\right)=N_{\bar{\alpha}\left(e_{j}\right)} \Rightarrow N_{\bar{\alpha}\left(e_{j-1}\right)}=N_{\bar{\alpha}\left(e_{j}\right)} \Rightarrow \bar{\alpha}\left(e_{j}\right)=$ $\bar{\alpha}\left(e_{j-1}\right)$. Repeating the process, we obtain $\bar{\alpha}\left(e_{j}\right)=\bar{\alpha}\left(e_{j-1}\right)=\cdots=\bar{\alpha}\left(e_{1}\right)=\bar{\alpha}\left(e_{0}\right)$. Thus by $(6.10)$

$$
\alpha\left(N_{e_{0}}\right)=\alpha\left(N_{e_{1}}\right)=N_{\bar{\alpha}\left(e_{0}\right)},
$$

and the proof is complete.

Lemma 6.3. Let $G, H$ be groups. Then every epimorphism $\alpha: G \rightarrow H$ is uniquely extended to a p epimorphism $\alpha^{*}: P(G) \rightarrow P(H)$ given by $x N \mapsto \alpha(x) \alpha(N)(x \in G, N \triangleleft G)$.

Proof. We have $P(G)=\bigcup_{N \in \mathbf{N}} G / N$ is a proper $q$ partial group with

$$
\varphi_{N, M}: G / N \longrightarrow G / M, \quad x N \longmapsto x M, \quad \text { if } N \geq M \text { (i.e., } N \subset M \text {, see Lemma 5.1), }
$$

since $\alpha$ is epimorphism, we have $\mathbf{N}(H)=\{\alpha(N): N \triangleleft G\}$ and it is easy to see that $\alpha^{*}$ : $P(G) \rightarrow P(H), x N \mapsto \alpha(x) \alpha(N),(x \in G, N \triangleleft G)$ is a well-defined epimorphism, and the restriction of $\alpha^{*}$ on $(P(G))_{1}=G$ is $\alpha: G \rightarrow H$. For any $N \triangleleft G$ we have by the definition of $\alpha^{*}, \alpha^{*} N=\alpha(N)$. Thus

$$
\alpha\left(\operatorname{ker} \varphi_{1, N}\right)=\alpha(N)=\operatorname{ker} \varphi_{1, \alpha(N)}=\operatorname{ker} \varphi_{1, \alpha^{*}} N
$$

Hence $\alpha^{*}$ is $p$ epimorphism. If $\beta^{*}: P(G) \rightarrow P(H)$ is a $p$ epimorphism, then by Lemma 6.1, the restriction of $\beta^{*}$ on $(P(G))_{1}=G$ defines an epimorphism $\beta: G \rightarrow H, x \mapsto x^{\prime}$, and for any $x N \in P(G)$, we have

$$
\beta^{*} x N=\beta(x) \beta^{*} N .
$$


Since $\beta$ is a $p$ epimorphism, we have

$$
\beta(N)=\beta\left(\operatorname{ker} \varphi_{1, N}\right)=\operatorname{ker} \varphi_{1, \beta^{*} N}=\beta^{*} N
$$

Thus, if $\beta=\alpha$, we have for any $N \triangleleft G$,

$$
\alpha(N)=\beta(N)=\operatorname{ker} \varphi_{1, \beta^{*} N}=\beta^{*} N .
$$

Therefore $\beta^{*}=\alpha^{*}$.

In view of Lemma 6.3, we may define an operation $*$ on epimorphisms of groups which sends every epimorphism $\alpha: G \rightarrow H$ to its unique extension $p$ epimorphism $\alpha^{*}$ : $P(G) \rightarrow P(H), x N \mapsto \alpha(x) \alpha(N)$. Some properties of $*$ is given in the following lemma, whose proof is trivial and omitted.

Lemma 6.4. (i) For any group $G, 1_{G}^{*}=1_{G}$.

(ii) Given a pair of epimorphisms $\alpha: G \rightarrow H$ and $\beta: H \rightarrow K$, then, $(\beta \alpha)^{*}=\beta^{*} \alpha^{*}$.

Given an epimorphism $\bar{\alpha}: S \rightarrow T$ of proper $q$ partial groups, its restriction $\alpha: S_{1} \rightarrow T_{1}$ is an epimorphism of groups (Lemma 6.1) and so extended uniquely to a $p$ epimorphism $\alpha^{*}: P\left(S_{1}\right) \rightarrow P\left(T_{1}\right), x N \mapsto \alpha(x) \alpha(N)$. Recall also that, for any proper $q$ partial group $S$, there is an isomorphism $\alpha_{S}: S \rightarrow P\left(S_{1}\right), x e \mapsto x N_{e}, x \in S_{1}, e \in E(S)$ (see Lemma 5.4, Theorem 5.6, and Corollary 5.9).

Lemma 6.5. Let $\bar{\alpha}: S \rightarrow T$ be an epimorphism of proper $q$ partial groups. Then $\bar{\alpha}$ is $p$ epimorphism if and only if $\bar{\alpha}=\alpha_{T}^{-1} \alpha^{*} \alpha_{S}$.

Proof. By Lemma 6.3, $\alpha^{*}$ is $p$ epimorphism. Thus for each $e \in E(S), \alpha^{*} x N_{e}=\alpha(x) \alpha\left(N_{e}\right)$. Suppose $\bar{\alpha}=\alpha_{T}^{-1} \alpha^{*} \alpha_{S}$, let $x \in S_{1}, e \in E(S)$, and let $\alpha\left(N_{e}\right)=N_{e^{\prime}}$ (for some $e^{\prime} \in E(S)$ ). Then, $\alpha(x) \bar{\alpha}(e)=\bar{\alpha}(x) \bar{\alpha}(e)=\bar{\alpha}(x e)=\alpha_{T}^{-1} \alpha^{*} \alpha_{S}(x e)=\alpha_{T}^{-1} \alpha^{*} x N_{e}=\alpha_{T}^{-1} \alpha(x) \alpha\left(N_{e}\right)=\alpha_{T}^{-1} \alpha(x) N_{e^{\prime}}$ $=\alpha(x) e^{\prime}$. Thus $\bar{\alpha}(e)=e^{\prime}$ ( since $\alpha(x) \in T_{1}$ ), and we have

$$
\alpha\left(\operatorname{ker} \varphi_{1, e}\right)=\alpha\left(N_{e}\right)=N_{e^{\prime}}=\operatorname{ker} \varphi_{1, e^{\prime}}=\operatorname{ker} \varphi_{1, \bar{\alpha}(e)} .
$$

Hence $\bar{\alpha}$ is $p$ epimorphism.

Conversely, if $\bar{\alpha}$ is $p$ epimorphism, then for all $e \in E(S), \alpha\left(N_{e}\right)=N_{\bar{\alpha}(e)}$. Thus for $x \in S_{1}$, $e \in E(S)$, we have

$$
\begin{aligned}
\alpha_{T}^{-1} \alpha^{*} \alpha_{S}(x e) & =\alpha_{T}^{-1} \alpha^{*} x N_{e}=\alpha_{T}^{-1}\left(\alpha(x) \alpha\left(N_{e}\right)\right) \\
& =\alpha_{T}^{-1}\left(\alpha(x) N_{\bar{\alpha}(e)}\right)=\alpha(x) \bar{\alpha}(e)=\bar{\alpha}(x e) .
\end{aligned}
$$

Therefore, $\alpha_{T}^{-1} \alpha^{*} \alpha_{S}=\bar{\alpha}$.

We notice that given $p$ epimorphisms $\bar{\alpha}: S \rightarrow T$ and $\bar{\beta}: T \rightarrow R, \bar{\beta} \bar{\alpha}: S \rightarrow R$ is a $p$ epimorphism (by the definition or Lemma 6.5). Also $1_{S}: S \rightarrow S, x \mapsto x$ is clearly $p$ epimorphism. Thus we can define a category:

PQP: objects are all proper $q$ partial groups $S, T, \ldots$, and morphisms $\operatorname{Hom}(S, T)=$ (possibly empty) set of all $p$ epimorphisms from $S$ onto $T$. 
Let $\mathbf{G}$ be the category of all groups with morphisms of all epimorphisms of groups. Thus for any two groups $G, H, \operatorname{Hom}(G, H)=$ (possibly empty) set of all epimorphisms from $G$ onto $H$.

Recall that two categories C, D are said to be equivalent if there exist functors

$$
F: \mathrm{C} \rightarrow \mathrm{D}, \quad G: \mathrm{D} \rightarrow \mathrm{C},
$$

and natural isomorphisms

$$
\eta: G F \cong I_{\mathrm{C}}, \quad \varepsilon: F G \cong I_{\mathrm{D}}
$$

where $I_{\mathrm{C}}$ and $I_{\mathrm{D}}$ are the identity functors.

Theorem 6.6. G and PQP are equivalent categories.

Proof. Define $P: \mathbf{G} \rightarrow$ PQP on objects by $G \mapsto P(G)$, where $P(G)$ is the proper $q$ partial group associated to $G$ (as in Lemma 5.1) and on morphisms by

$$
\alpha: G \longrightarrow H \longmapsto \alpha^{*}: P(G) \longrightarrow P(H)
$$

where $\alpha^{*}$ is the unique $p$ epimorphism extension of $\alpha$ on $P(G)$, defined as in Lemma 6.3. By Lemma 6.4, $P$ is a functor (actually is full and faithful). Define $R: \mathbf{P Q P} \rightarrow \mathbf{G}$ on objects by $S \mapsto S_{1}$, and on morphisms by

$$
\bar{\alpha}: S \longrightarrow T \longmapsto \alpha: S_{1} \longmapsto T_{1},
$$

where $\alpha$ is the restrection of $\bar{\alpha}$ on $S_{1}$ (see Lemma 6.1). Clearly $R$ is a functor and $R P=I_{\mathrm{G}}$. In other words, there exists a natural isomorphism $\eta: R P \cong I_{\mathrm{G}}$ given by for any object $G$ in $\mathbf{G}, R P(G)=G$ and $\eta_{G}: R P(G) \rightarrow G$ is the identity $1_{G}, x \mapsto x$. Next for each object $S$ in PQP, $P R(S)=P\left(S_{1}\right)$. Define $\varepsilon_{S}: P R(S) \rightarrow I(S)$, that is, $\varepsilon_{S}: P\left(S_{1}\right) \rightarrow S$ by $x N_{e} \mapsto x e$, that is, $\varepsilon_{S}=\alpha_{S}^{-1}$. Thus $\varepsilon_{S}$ is an invertible (isomorphism) in PQP and for any morphism $\bar{\alpha}: S \rightarrow T$ in $\mathbf{P Q P}$ we have a diagram

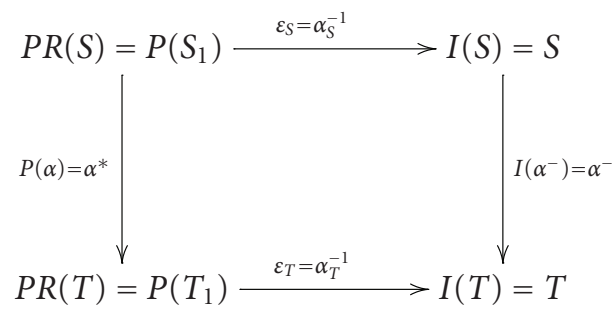

Applying Lemma 6.5, we have

$$
\bar{\alpha} \varepsilon_{S}=\bar{\alpha} \alpha_{S}^{-1}=\left(\alpha_{T}^{-1} \alpha^{*} \alpha_{S}\right) \alpha_{S}^{-1}=\alpha_{T}^{-1} \alpha^{*}=\varepsilon_{T} \alpha^{*},
$$

and the above diagram is commutative. This gives a natural isomorphism $\varepsilon: P R \cong I$ whose components are $\varepsilon_{S}=\alpha_{S}^{-1}$ and the proof is complete. 


\section{References}

[1] A. M. Abd-Allah and M. E.-G. M. Abdallah, Categories of generalized rings associated with partial mappings, Pure Mathematics Manuscript 7 (1988), 53-65.

[2] __ Congruences on Clifford semigroups, Pure Mathematics Manuscript 7 (1988), 19-35.

[3] _ On Clifford semigroups, Pure Mathematics Manuscript 7 (1988), 1-17.

[4] _ Semilattices of monoids, Indian Journal of Mathematics 33 (1991), no. 3, 325-333 (1992).

[5] J. M. Howie, An Introduction to Semigroup Theory, L.M.S. Monographs, no. 7, Academic Press, London, 1976.

[6] T. W. Hungerford, Algebra, Graduate Texts in Mathematics, vol. 73, Springer, New York, 1980.

[7] S. Mac Lane, Categories for the Working Mathematician, Graduate Texts in Mathematics, vol. 5, Springer, New York, 1971.

[8] M. Petrich, Inverse Semigroups, Pure and Applied Mathematics (New York), John Wiley \& Sons, New York, 1984.

[9] D. J. S. Robinson, A Course in the Theory of Groups, Graduate Texts in Mathematics, vol. 80, Springer, New York, 1982.

M. El-Ghali M. Abdallah: Department of Mathematics, Faculty of Science, Menoufiya University, Shebin El-Kom 32511, Egypt

E-mail address: mohamed_elghaly@yahoo.com

L. N. Gab-Alla: Department of Mathematics, Faculty of Science, Menoufiya University,

Shebin El-Kom 32511, Egypt

E-mail address: layla_nashed2006@yahoo.com

Sayed K. M. Elagan: Department of Mathematics, Faculty of Science, Menoufiya University,

Shebin El-Kom 32511, Egypt

E-mail address: sayed_khalil2000@yahoo.com 


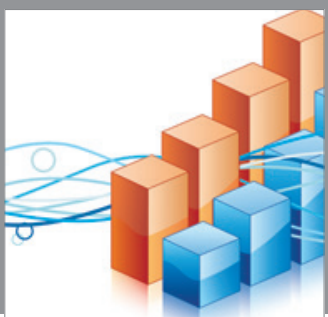

Advances in

Operations Research

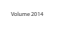

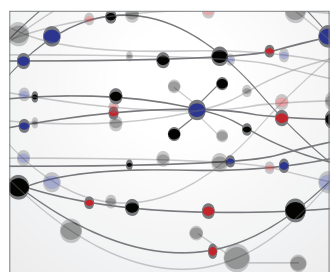

\section{The Scientific} World Journal
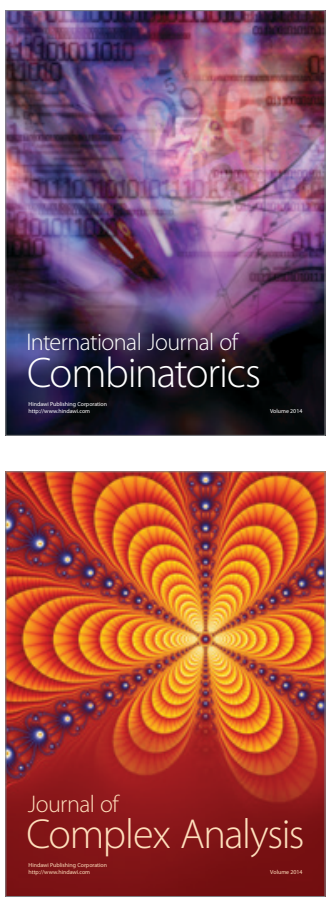

International Journal of

Mathematics and

Mathematical

Sciences
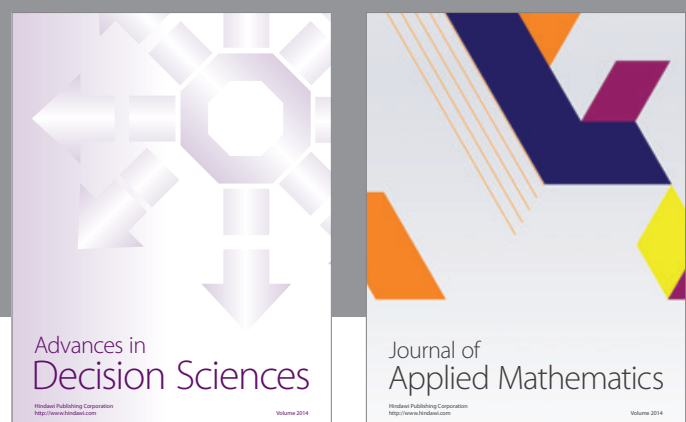

Journal of

Applied Mathematics
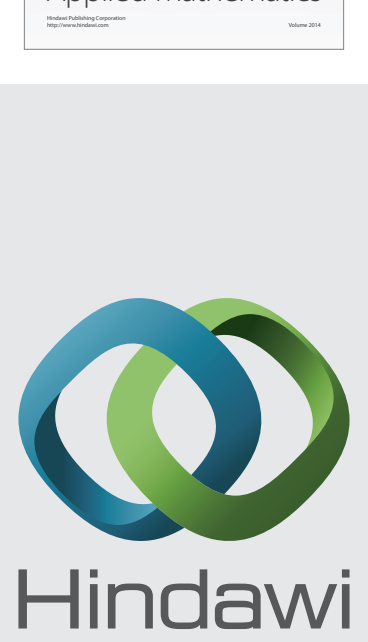

Submit your manuscripts at http://www.hindawi.com
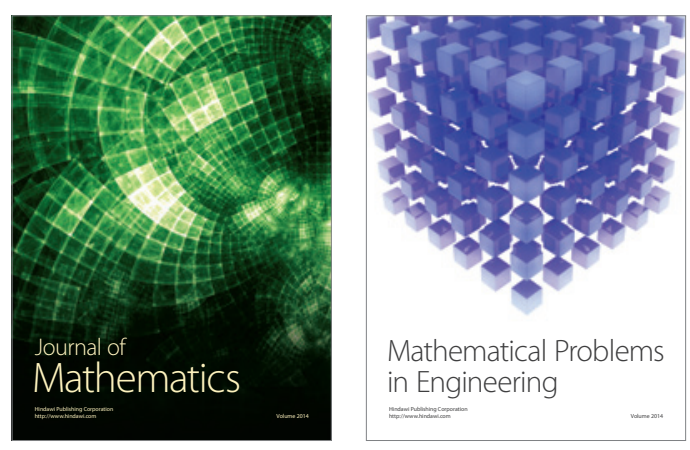

Mathematical Problems in Engineering
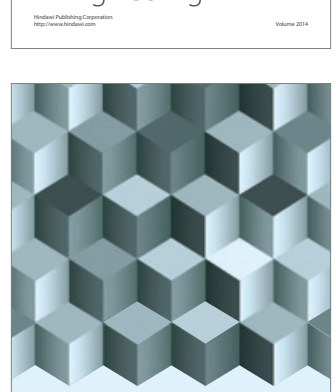

Journal of

Function Spaces
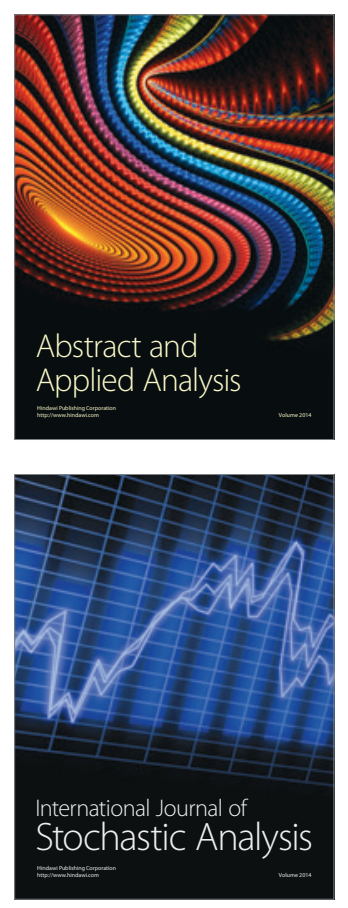

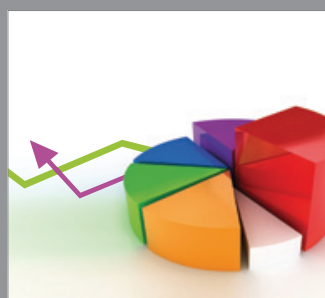

ournal of

Probability and Statistics

Promensencen
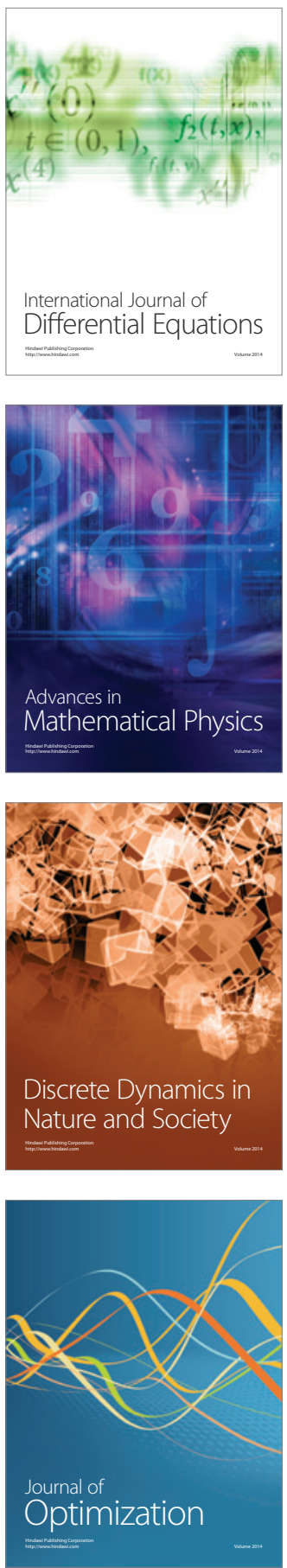